\title{
Sprachliche Zweifelsfälle als linguistischer Gegenstand. \\ Zur Einführung in ein vergessenes Thema der Sprachwissenschaft.*
}

\author{
Wolf Peter Klein (Berlin)
}

\begin{abstract}
The term "sprachlicher Zweifelsfall" ('linguistic case of doubt') is not yet well established in the terminology of linguistics. The first part of the paper therefore outlines a definition of "sprachlicher Zweifelsfall" and a classification of various types. Basically, the definition takes up the fact that even fully competent speakers sometimes do not know definitely which variant to choose from two (or more) alternatives. Doubts like this evolve on all levels of language (phonetics, morphology, lexic, syntax, semantics). Considering the emergence of "Zweifelsfälle", it is argued that they arise mainly from the existence of written language and more or less standardized varieties of language. At least some of their features refer to the specific conceptual conditions of writing and reading. In the second part of the paper, the "Zweifelsfälle" are reconceived in so far as they have been treated in the newer history of German discourse on language, including both popular and philological discussion. Starting in 19th century thought, they became symbolic means of social distinction. In contrast, linguists tended to neglect the existence of "Zweifelsfälle" because they did not fit in well with their theories on language. The paper ends with several theses dealing with possible future research on the topic.
\end{abstract}

\section{$1 \quad$ Einführung}

Sucht man in den gängigen linguistischen Fachlexika nach Rat, was wohl der Begriff "sprachlicher Zweifelsfall" bedeutet, so wird man enttäuscht. Ein entsprechendes Lemma fehlt (z.B. Glück 2000, Bußmann 2002). Schon angesichts dieses lexikographischen Befundes lässt sich wohl behaupten, dass der Begriff des "sprachlichen Zweifelsfalls" in der heutigen Linguistik keine oder höchstens eine marginale Rolle spielt. Lediglich in sprachhistorischen Zusammenhängen begegnet man ihm mit etwas höherer Frequenz, bei der allerdings längst nicht alle Facetten seiner Bedeutung zum Tragen kommen (Klein 2000: 70f). Für einen Themenband, in dessen Titel der Begriff an prominenter Stelle auftaucht, resultieren aus diesem Zustand einige grundsätzliche Fragen und Probleme, darunter beispielsweise die folgenden: Was ist überhaupt ein Zweifelsfall? Welche Typen können in einer theoretischen Grund-

\footnotetext{
* Im Zuge der anonymen Begutachtung dieses Aufsatzes für Linguistik online wurden mir viele hilfreiche Verbesserungshinweise mitgeteilt. Dankeschön! (WPK)
} 
satzüberlegung unterschieden werden? Warum gibt es überhaupt Zweifelsfälle? Wie wurden sie bisher behandelt?

Dieser Aufsatz will sich derartigen elementaren Fragen widmen. Das Ziel liegt also darin, im Lichte der linguistischen Vernachlässigung von sprachlichen Zweifelsfällen das Themenfeld des Bandes grob abzustecken. Der angesprochene Horizont hat einige Folgen für den Status der hier präsentierten Überlegungen. Da es sich nämlich um die erste Sondierung eines weiten Terrains handelt, tauchen einzelne Zweifelsfälle nur zur Illustration theoretischterminologischer Reflexionen auf. In keinem Fall wurde auch nur von Ferne angestrebt, einen einzelnen Zweifelsfall erschöpfend zu analysieren. Der einführende Charakter bringt es zudem mit sich, dass einige Selbstverständlichkeiten thematisiert werden müssen. So trivial dies manchem linguistischen Leser erscheinen mag, so unvermeidbar ist dies, wenn man in einigen Grundsatzüberlegungen einen bisher wenig explizierten Begriff für die weitere Diskussion klären möchte. Ohnehin ist es ja gefährlich, in wissenschaftlichen Dingen manche Sachen für selbstverständlich zu halten.

Ich möchte meine Überlegungen in zwei großen Teilen ausformulieren. Der erste Teil (I.) ist der Theorie und Terminologie der Zweifelsfälle gewidmet. Der zweite Teil (II.) thematisiert die metasprachliche Geschichte und Gegenwart der Zweifelsfälle im Deutschen. In einigen Thesen wird der Inhalt am Ende in bestimmten Perspektiven zugespitzt. Dabei soll, dem Thema des Aufsatzes entsprechend, vor allem auf die linguistische Behandlung der Zweifelsfälle abgehoben werden.

\section{Theorie und Terminologie der Zweifelsfälle}

Theoretisch orientierte und empirisch bewusste Überlegungen zur Relevanz eines Fachworts sind in mehreren Richtungen anzustellen. Zunächst muss es um die Definition des jeweiligen Begriffs und die methodologischen Identifikationsmöglichkeiten der bezeichneten Erscheinung, dann um eine vorläufige typologisch-genetische Klassifikation des zugehörigen Phänomenbereichs gehen. Im vorliegenden Fall soll noch hinzukommen, dass der Begriff des sprachlichen Zweifelsfalls eine eminent praktische Bedeutung besitzt. Denn Zweifelsfälle setzen in der Sprachwirklichkeit und -wissenschaft nicht nur (theoretische) Reflexionen in Gang, sondern sie werfen auch praktische Probleme auf, die ganz nüchterne Antworten erfordern. Entsprechend möchte ich in diesem Kapitel vier Problemkomplexe in den Mittelpunkt stellen und in den folgenden Abschnitten näher erörtern:

1. Definition: Wie kann der Begriff "sprachlicher Zweifelsfall" definiert werden?

2. Identifikation: Wie lassen sich auf der Basis der gegebenen Definition Zweifelsfälle empirisch identifizieren?

3. Klassifikation: Was gibt es für Klassen von Zweifelsfällen und worin wurzelt ihre Entstehung?

4. Klärung: Mit welchen Typen von Antworten lassen sich Zweifelsfälle praktisch "klären"? 


\subsection{Definition}

Ich schlage eine Definition vor, in der auf der Basis des Sprachverhaltens realer Sprecher bestimmte Formulierungspaare als konstitutive Bestandteile von Zweifelsfällen identifiziert werden. Anders gesagt, aus der konkret erlebten Sprachwirklichkeit der Sprecher sollen durch die Ansetzung bestimmter definitorischer Gehalte sprachliche Einheiten herausgefiltert und so als Zweifelsfälle bezeichnet werden. Durch dieses Verfahren können auf Zweifelsfälle sowohl systemlinguistische als auch pragmatische Untersuchungsperspektiven angewandt werden. Die Definition lautet:

Ein sprachlicher Zweifelsfall (Zf) ist eine sprachliche Einheit (Wort/Wortform/Satz), bei der kompetente Sprecher (a.) im Blick auf (mindestens) zwei Varianten (a, b...) in Zweifel geraten (b.) können, welche der beiden Formen (standardsprachlich) (c.) korrekt ist (vgl. Sprachschwankung, Doppelform, Dublette). Die beiden Varianten eines Zweifelsfalls sind formseitig oft teilidentisch (d.) (z.B. dubios/dubiös, lösbar/löslich, des Automat/des Automaten, Rad fahren/rad fahren/radfahren, Staub gesaugt/staubgesaugt/gestaubsaugt).

Zur näheren Erläuterung sollen im folgenden die unterstrichenen Passagen der Definition etwas genauer geklärt werden, um so die verschiedenen Facetten des Begriffs und ihre Zusammenhänge - zumindest phänomenologisch - zu beleuchten. Sofern möglich, werden dabei auch einige Konsequenzen für die linguistische Thematisierung der Zweifelsfälle angedeutet.

a.) Kompetente Sprecher: Als Ausgangspunkt der Definition werden die voll kompetenten Sprecher und ihr Sprachverhalten gewählt. ${ }^{1}$ Sie sind die Subjekte des Zweifelns, das hier definitorisch in den Blick genommen wird. Es sind also nicht die Sprachwissenschaftler, die möglicherweise über die angemessene Analyse eines bestimmten sprachlichen Phänomens ins Grübeln geraten und so Zweifelsfälle identifizieren könnten. Daraus folgt auch, dass die Debatten und Inkompatibilitäten zwischen den verschiedenen linguistischen Schulen und Diskussionskontexten für die Begriffsdefinition keine Rolle spielen. Auch geht es nicht um (sprachlich in- oder teilkompetente) Personen, deren Spracherwerb als muttersprachliche oder fremdsprachliche Lerner noch nicht abgeschlossen ist und die von daher zweifeln oder unkorrekt sprechen mögen.

Der Bezug auf kompetente Sprecher dürfte eine Möglichkeit bieten, in einem ersten Schritt den Begriff des "Zweifelsfalls" eindeutig vom Begriff des "sprachlichen Fehlers"2 zu differenzieren. Auch kompetente Sprecher machen natürlich "Fehler", etwa in kritischen Perfor-

\footnotetext{
1 Zu den Eigenschaften, die den "kompetenten Sprecher" methodologisch als Bezugsgröße in der Linguistik auszeichnen, vgl. z.B. Grewendorf et al. 1990:32f.

2 Die bisherigen Arbeiten zur sog. Fehlerlinguistik gingen meistens von Situationen des Fremdspracherwerbs aus und sind von daher für eine Definition, die kompetente muttersprachliche Sprecher in den Mittelpunkt stellt, höchstens mittelbar relevant (z.B. Kielhöfer 1980, im didaktischen Überblick Kleppin 1998). Eine Ausnahme dazu bilden vor allem einige Aufsätze zum Thema in Cherubim 1980.
} 
manzsituationen ("Sprechlapsus", "Versprecher") 3 oder dann, wenn ihre Sprachkompetenz (noch) bestimmte Lücken aufweist. Derartige Abweichungen vom Usus kommen keineswegs immer zufällig zustande, sondern ergeben sich aufgrund bestimmter Bedingungen, die der linguistischen Analyse zugänglich sind. Der Unterschied zu den Zweifelsfällen liegt darin, dass kompetente Sprecher, nachdem sie einen Performanzfehler oder einen Versprecher produziert haben, die jeweilige Formulierung rückblickend auch als (grammatisch) falsch, da nicht im Usus verankert, bezeichnen würden, während bei Zweifelsfällen auch im etwaigen Rückblick unklar bleibt, ob die jeweils andere Variante nicht auch hätte korrekt sein können. ${ }^{4}$ In dieser Festlegung mag zwar noch ein kleiner Bereich der Unklarheit übrig bleiben, in dem bestimmte Phänomene sowohl als Fehler als auch als Zweifelsfälle zu klassifizieren wären. Gleichwohl dürfte das genannte Unterscheidungskriterium in sehr vielen Situationen eine eindeutige Trennung zwischen Zweifelsfällen und Fehlern bzw. Versprechern gewährleisten. Auf jeden Fall ist es wichtig, die klare Unterscheidung zwischen Zweifelsfällen und sprachlichen Formen von Defektivität (Fehler, Versprecher) nicht aus den Augen zu verlieren.

In der Definition drückt sich demnach die Tatsache aus, dass in der Sprachwirklichkeit selbst Zweifel vorkommen. Sie wären auch dann vorhanden, wenn es gar keine Sprachwissenschaft gäbe, die sich ihrer Existenz annehmen würde. Ich gehe also davon aus, dass kompetente Sprecher sich gelegentlich in Situationen wieder finden, in denen sie nicht genau wissen, welche von (mindestens) zwei Formulierungen die richtige ist. Auf die pluralische Form des Zweifelssubjekts - die Sprecher statt der Sprecher/die Sprecherin - möchte ich an dieser Stelle nur insofern hinweisen, als dass damit eine linguistische Selbstverständlichkeit verbunden ist. Natürlich kann nicht jeder Zweifel eines Individuums Anlass dafür sein, sprachwissenschaftlich von einem Zweifelsfall zu sprechen. Es muss vielmehr deutliche Hinweise darauf geben, dass der jeweilige Fall nicht nur einen, zwei oder drei Sprecher in Zweifel stürzen kann, sondern im Rahmen einer Sprachgemeinschaft tatsächlich ein mehr oder weniger allgemeines Phänomen darstellt. Im Rahmen der Überlegungen zur Identifikationsproblematik (=2.) ist auf diesen Punkt zurück zu kommen.

b.) In Zweifel geraten: Im Zweifel des kompetenten Sprechers über ein Wort, eine Wortform oder einen Satz drückt sich ein Moment des Innehaltens aus, das bei der Untersuchung von Zweifelsfällen von besonderer Bedeutung ist. Man zweifelt über die Korrektheit eines sprachlichen Phänomens und erhebt in dieser gedanklichen Bewegung Sprache zu einem Reflexionsgegenstand. Das soll natürlich nicht heißen, dass hier stets kleine Linguisten am Werke sind. Vielmehr möchte ich nur darauf hinweisen, dass in jedem Sprachzweifel Sprache vergegenständlicht wird und, zumindest in ersten Ansätzen, als bewusstes Objekt von Überlegungen gefasst wird. Daraus folgt: Zweifelsfälle sind immer mit einem, wenn auch nur rudimentären, metasprachlichen Bewusstsein verbunden. Negativ formuliert: Sie sind quasi Stolpersteine in der natürlichen Kommunikation, die zum Ausgangspunkt von sprachreflexiven

\footnotetext{
3 Zum Problem der "Versprecher" vgl. z.B. Leuninger 1998, wegweisend für die Forschung war die Sammlung von Fromkin 1980, siehe auch Bierwisch 1981.

${ }^{4}$ Fehler und Versprecher sind insofern mehr oder weniger problemlos mit dem sprachtheoretisch einschlägigen "Prinzip der klaren Fälle" (Chomsky) zu konzeptualisieren, während das bei Zweifelsfällen nicht so einfach möglich ist, vgl. immer noch sehr erhellend Reis 1979: 4ff.
} 
Gedanken und Äußerungen werden können. In der Verquickung von natürlichem Sprachverhalten und metasprachlicher Reflexion, in der Sprache mit weitreichenden Folgen vergegenständlicht wird, liegt ein Gehalt, an dem das öffentliche und individuelle Sprachbewusstsein mit der linguistischen Gegenstandskonstitution in Berührung kommt (Klein 1986:18-23).

Zweifelsfälle bieten demnach generelle Anlässe zum Nachdenken über Sprache und lassen sich insofern auch im Rahmen übergreifender gesellschaftlicher Bewegungen, in denen Sprache und Kommunikation thematisiert wird, für bestimmte Zwecke instrumentalisieren. Einschlägig ist hier beispielsweise das Sprachbewusstsein, das in der Schule vermittelt und in bildungssprachlichen Sprachdebatten und Stellungnahmen immer wieder aktualisiert wird. Die Existenz von Zweifelsfällen hat zur Folge, dass in einer Sprachgemeinschaft Institutionen und Personen tätig werden (müssen?), die in Zweifelsfällen orientierend und normierend wirken oder die Existenz von Zweifelsfällen in anderer Art und Weise aufgreifen und thematisieren. Dies kann beispielsweise durch sprachbezogene Publikationen (z.B. populäre Grammatiken, "Sprachverbesserungsbücher", Stillehren, Briefsteller), Sprachunterricht, Sprachdiskussion und Sprachberatung erfolgen. Im Teil II des Aufsatzes werde ich auf diese Dimension im Rahmen der jüngeren deutschen Sprachgeschichte zurückkommen.

c.) Standardsprachlich: In der Regel gilt der Zweifel der Frage, inwiefern ein sprachliches Phänomen standardsprachlich als korrekt bzw. unkorrekt zu gelten hat. Das ist nicht selbstverständlich. Denn hier wären durchaus auch andere Zielpunkte denkbar. Beispielsweise ist es nicht ausgeschlossen, dass zweifelnde Sprecher als Fixpunkt einen Dialekt, eine Fachsprache oder eine bestimmte Stillage thematisieren. Das dürfte aber eher selten der Fall sein. Schließlich nutzen die Sprecher in derartigen Fällen oft (vergegenständlichende, s.o.) Zielbegriffe wie "gutes Deutsch", "richtiges Deutsch" oder "normales Deutsch", also Begriffe, in denen etwaige Varietäten gerade nicht in den Blick kommen, sondern von einer - wie auch immer gearteten - einheitlichen deutschen Sprache ausgegangen wird. Als Bewusstseinsbegriff besitzt die Standardsprache also eine ganz konkrete Realität, insofern die Sprecher sie immer wieder als Entscheidungsinstanz in Zweifelsfällen geltend machen. ${ }^{5}$ Möglicherweise ist der alltagssprachliche Begriff der deutschen Standardsprache ("gutes Deutsch" u.a.) sogar genealogisch aus Situationen entstanden, in denen Sprecher über Zweifelsfälle reflektieren. Im Nachdenken über mögliche Varianten und dem kommunikativen Druck, sich für eine der Möglichkeiten entscheiden zu müssen, ${ }^{6}$ muss notwendigerweise (!?) eine Instanz Gestalt annehmen, die (implizit oder explizit) als argumentative Rechtfertigung ("Standard") für die Auswahl bestimmter Varianten fungiert. Wer von Zweifelsfällen spricht, sollte daher von der

\footnotetext{
${ }^{5}$ Ich betone diesen Punkt auch deshalb, weil es in der (grammatischen) Literatur immer wieder Stellungnahmen gibt, die die Standardsprache als bloßes von den Linguisten geschaffenes (oder "idealisiertes") "Abstraktum" oder "Konstrukt" ansehen (z.B. Engel 1988:A006; Grewendorf et al. 1990:35). Demgegenüber gehe ich davon aus, dass dem Begriff der Standardsprache durchaus ein identifizierbares nicht-triviales Substrat in der Sprachwirklichkeit entspricht.

${ }^{6}$ Man kann schließlich in natürlicher Kommunikation nicht zwei Varianten gleichzeitig benutzen.
} 
Standardsprache und allen damit verbundenen linguistischen und sprachpraktischen Problemen nicht schweigen.

Die Standardsprache - oder genauer: das jeweilige Bewusstsein von Standardsprache - spielt darüber hinaus sogar in verschärfter Form eine große Rolle bei den zweifelnden Sprechern. Denn oft gehen sie von der Existenz einer explizit normativ geregelten oder zumindest regelbaren Standardsprache aus, die als Klärungsinstanz bei Varianten fungieren soll - und dies nicht allein auf dem Feld der Rechtschreibung. Die sprachlichen Zweifelsfälle sind also nicht in ein neutrales metasprachliches Bewusstseinfeld integriert, sondern in ein stark normativ geprägtes. Auch in diesem Kontext ist wieder auf die große Bedeutung des schulisch vermittelten Sprachbewusstseins sowie auf die öffentliche Sprachdiskussion hinzuweisen. Zusammenfassend werde ich insofern von normativem Sprachbewusstsein sprechen. An der Schnittstelle zwischen der objekt- und der metasprachlichen Dimension der Zweifelsfälle harren viele Themen der genaueren Erforschung. Es wäre beispielsweise zu prüfen, ob und, wenn ja, wie möglichlicherweise Destandardisierungserscheinungen (Mattheier 1997) und ein abgeschwächtes Normverständnis (Hinweise bei Davies 2001, Beck 1996) die Existenz und das hergebrachte Bewusstsein von Zweifelsfällen in der deutschen Sprachgemeinschaft der Gegenwart verändern. Sprachhistorisch könnte untersucht werden, inwiefern die metasprachliche Thematisierung von Zweifelsfällen die objektsprachliche Entwicklung des Deutschen möglicherweise entscheidend beeinflusst hat.

d.) Formseitig oft teilidentisch: Die beiden Varianten eines Zweifelsfalls haben, was ihre Ausdrucksseite angeht, meistens eine charakteristische Beziehung. Sie unterscheiden sich nämlich meistens nur in Details. Zur Illustration sollen außer den in der Definition bereits aufgelisteten Beispielen noch einige weitere angeführt werden: Heißt es minus Rabatt oder minus des Rabatts? Stellt Friede oder Frieden die korrekte Wortform dar? Gehört in das Kompositum Kriegführung ein Fugen-s? Muss im Genitiv Kindes oder Kinds gesagt werden? Als Hintergrundannahme steht bei solchen Fragen oft die Annahme der Sprecher im Raum, dass unterschiedliche sprachliche Formen stets auch unterschiedliche Bedeutungen haben bzw. Funktionen erfüllen müssen. ${ }^{7}$ In letzter Konsequenz orientieren sich die Sprecher dabei stets an Identitäts- bzw. Differenzurteilen, wenn sie die teilidentischen Varianten eines Zweifelsfalls in den Blick nehmen: Gibt es zwischen den Varianten a und b einen (semantischen) Unterschied oder handelt es sich um Äquivalente? Wenn es keinen Unterschied gibt, warum existieren dann überhaupt die Varianten? Könnte man mutmaßlich synonymen Varianten nicht doch unterschiedliche Funktionen oder Bedeutungen zuschreiben?

Das Merkmal der formseitigen Teilidentität ist freilich kein hinreichendes Kriterium für das Vorliegen eines Zweifelsfalls. Denn keineswegs alle Wortpaare, die sich nur in Details unterscheiden, sind ja zu den Zweifelsfällen zu rechnen. Niemand wird beispielsweise ernsthaft darüber grübeln, ob es der Käse oder der Käsen heißt, ob des Lesers (Gen.) oder des Leseres richtiges Deutsch ist oder ob der Plural von einem Wort wie Tiger mit $-s$ gebildet wird oder

\footnotetext{
7 Diese Annahme ist tief in der Phase des Erstspracherwerbs verankert und als "Prinzip des Kontrasts" terminologisch bereits einschlägig erfasst: "Sprecher nehmen an, daß Unterschiede hinsichtlich der Form auch Unterschiede in der Wortbedeutung signalisieren." (Meibauer 1999:171).
} 
nicht. Schließlich gehören auch (phonologische, graphematische) Minimalpaare (Typ: Gasse/Kasse) eindeutig nicht zu den Zweifelsfällen. Aus einer anderen Perspektive sprechen diese Befunde dafür, dass für beide Varianten eines Zweifelsfalls jeweils Faktoren angegeben werden können, die für ihre Grammatikalität sprechen. Dagegen ist bei Fehlern und Versprechern die eine Variante immer zweifellos richtig, die andere dagegen zweifellos falsch; bei Minimalpaaren sind beide Ausdrücke zweifellos richtig.

Ausgehend von derartigen formorientierten Überlegungen kommt eine wichtige Aufgabe bei der Erforschung von Zweifelsfällen in den Blick: Die Linguistik muss bei den Varianten eines Zweifelsfalls nämlich zwei dynamisch miteinander verbundene Faktoren identifizieren, wenn sie ihren sprachlichen Status angemessen analysieren möchte: sowohl die Faktoren, die für die Grammatikalität der jeweiligen Variante sprechen, als auch diejenigen, die ihre Ungrammatikalität rechtfertigen. Oft wird es dabei hilfreich sein, nicht mit Dichotomien, sondern mit skalierenden und graduierenden Begriffen zu arbeiten und im Sinne einer realistischen Grammatik einen Bereich der "Unbestimmtheit" (Reis 1978) anzusetzen. So könnte auch der Begriff der Ungrammatikalität durch den der grammatischen Randständigkeit oder des periphären Systemstatus ersetzt werden. Gelingt es, Faktoren auf diesen beiden Ebenen dingfest zu machen, so kann der schwebende Status der Zweifelsfälle analytisch eindeutig erfasst werden, insofern ihre Varianten in ein Spannungsfeld verschiedener Regularitäten eingeordnet wurden und dadurch ihr spezifisches Profil erklärbar wird. In diesem Sinn kann die Linguistik auf der einen Seite klären helfen, warum Sprecher an bestimmten Punkten überhaupt immer wieder zweifeln, auf der anderen Seite kann aus einer solchen Klärung hilfreiche und praktische Orientierung in Zweifelsfällen resultieren.

Zum Abschluss des definitorischen Teils soll der Begriff des sprachlichen Zweifelsfalls kurz im Rahmen der gängigen theoretischen Grundlegungen der Sprachwissenschaft betrachtet werden. Viele Linguisten gehen heutzutage im Anschluss an Chomsky davon aus, es sei Aufgabe der Sprachwissenschaft, die Kompetenz der Sprecher zu rekonstruieren (z.B. Grewendorf et al. 1990:31ff, Coseriu 1988). Man kann insofern von kompetenztheoretischen Fundierungen oder - einfacher - von Kompetenzlinguistik sprechen.

Obwohl oben ebenfalls mit dem Konzept des kompetenten Sprechers gearbeitet wurde, passen die Zweifelsfälle aber eigentlich nicht gut in das hergebrachte Konzept kompetenztheoretischer Fundierungen. Zum einen ist bei ihren Varianten nicht einfach zu entscheiden, ob sie grammatisch oder ungrammatisch sind. Die Zweifelsfälle sind in einer Grauzone angesiedelt, in der die Trennung zwischen Grammatikalität und Ungrammatikalität nicht wie üblich funktioniert. Zudem zeugen die sprachlichen Zweifelsfälle nicht von einem sprachlichen Können bzw. Wissen, sondern gerade vom Gegenteil: Man weiß ja gerade nicht, welche Variante der Zweifelsfälle korrekt ist. Sie sind nicht in der problemlos ablaufenden Kommunikation angesiedelt, die sinnvollerweise kompetenztheoretisch greifbar ist. Stattdessen zeugen sie vom Nicht-Können, also von den Momenten, in denen ein simpler Rückgriff auf sprachliche Kompetenz gerade nicht gelingt, weil der übliche kommunikative Fluss stockt und das jeweilige Hindernis als Zweifelsfall metasprachlich zu Bewusstsein kommt. Sicherlich etwas überspitzt könnte man sagen, dass Zweifelsfälle nur in einem Konzept greifbar werden, in dem neben 
der sprachlichen Kompetenz auch die sprachliche Inkompetenz (bzw. "Imperfektibilität" (Antos 1996: I, Kap. 3)) vorgesehen ist. Derart könnte die Reflexion über sprachliche Zweifelsfälle zu einer realistischen Umorientierung des traditionellen Kompetenzbegriffs beitragen: Zu jeder sprachlichen Kompetenz gehören notwendigerweise (!) Bereiche der Inkompetenz, zumindest der unsicheren Kompetenz (vgl. dazu auch unten Kap. 5).

Im Lichte dieser Bestimmungen ließe sich sogar behaupten, dass die Zweifelsfälle quasi den Skandal der modernen Sprachwissenschaft repräsentieren. Sie scheinen offensichtlich nicht gut ins theoretische Konzept zu passen. Die Vernachlässigung der Zweifelsfälle in der modernen Linguistik wird vor diesem Hintergrund etwas verständlicher, gerechtfertigt ist sie dadurch sicher nicht. Überdies wirft die Konstellation ein Licht auf die oft beklagte Spannung zwischen öffentlicher Sprachdiskussion und wissenschaftlicher Sprachforschung (z.B. Dieckmann 1991, Jäger 1999, Niederhauser 1999): Genau das, was in der nicht-linguistischen Sprachreflexion immer wieder Anlässe für metasprachliches Raisonnieren abgibt, wird in den sprachtheoretischen Grundlegungen der Kompetenzlinguistik tendenziell wegtheoretisiert. Von daher ist es eigentlich nicht verwunderlich, dass es eher selten zu einem fruchtbaren Austausch zwischen der Linguistik und den sprachinteressierten Kreisen der Öffentlichkeit kommt.

\subsection{Identifikation}

Die gerade angestellten Überlegungen gestatten uns, theoretisch näher einzukreisen, welche linguistischen Gehalte mit dem Begriff "sprachlicher Zweifelsfall" verbunden sind und über welche Eigenschaften Wortpaare verfügen müssen, wenn sie als sprachlicher Zweifelsfall bezeichnet werden sollen. Zu einer Theorie der sprachlichen Zweifelsfälle gehört neben dieser definitorischen Perspektive eine methodologische Überlegung zu dem Problem, wie sich auf der Basis einer solchen Festlegung Zweifelsfälle empirisch identifizieren lassen: Wie lässt sich klären, über welche sprachlichen Formen in einer Sprachgemeinschaft in einem hier relevanten Sinn tatsächlich gezweifelt wird?

Dabei ist, wie oben schon angesprochen, darauf zu achten, dass nicht jeder individuelle Akt des Zweifelns über Sprache zur Folge haben kann, dass das jeweilige Objekt als Zweifelsfall kategorisiert wird. Umgekehrt ist aber auch nicht voraus zu setzen, dass ein Zweifelsfall stets bei allen Sprechern als solcher begriffen wird. Zweifelsfälle sind ja nur potentielle Anknüpfungspunkte für metasprachliches Reflektieren. Für die empirische Identifikation von Zweifelsfällen muss vielmehr sichergestellt sein, dass sie bei vielen (nicht: allen, einzelnen) Sprechern und insofern immer wieder thematisiert werden.

Im Blick auf die Tatsache, dass Zweifelsfälle immer aus Varianten bestehen, muss ferner auf eine weitere methodologische Komplikation hingewiesen werden. Allein die Feststellung von Varianten reicht nämlich nicht aus, um ein Wortpaar als Zweifelsfall zu identifizieren. Denn dazu gehört der Nachweis, dass über dieses Wortpaar auch in relevanter Art und Weise (metasprachlich) reflektiert wird. Neben Varianten, über die die Sprachgemeinschaft immer wieder nachdenkt, mag es nämlich durchaus Varianten geben, die kaum zu einem einschlägigen ge- 
danklichen Gegenstand erhoben werden. Definitionsgemäß müssen Zweifelsfälle aber über eine gewisse metasprachliche Komponente verfügen.

Ich möchte sechs Möglichkeiten festhalten, wie für ein gegebenes Sprachstadium Hinweise auf die Existenz von Zweifelsfällen festgestellt werden können. Folgende Fälle lassen sich unterscheiden:

a. Durchsicht der Datenbanken und Erfahrungsberichte von Sprachberatungsstellen,

b. Durchsicht bereits existierender Zweifelsfallsammlungen (resultieren oft aus a.),

c. Analyse natürlicher metasprachlicher Thematisierungen von Zweifelsfällen,

d. Umfragen/empirische Erhebungen zur Existenz von Zweifelsfällen,

e. Analyse von Hinweisen auf Zweifelsfälle in existierenden Grammatiken und Wörterbüchern,

f. Befragung der eigenen Sprachkompetenz ("Sprachgefühl").

Die sechs Möglichkeiten sollen an dieser Stelle nicht eingehend erläutert werden. Gleichwohl möchte ich zur methodologischen Problematik der Identifikation von Zweifelsfällen einige Bemerkungen machen: Die einfachste Möglichkeit stellt vermutlich die Variante a., mittelbar auch b. dar. Es gibt diverse Erfahrungsberichte von Sprachberatungsstellen, die auch Hinweise auf existierende Datenbanken enthalten. Hier seien nur entsprechende Darstellungen und Materialsammlungen zur DUDEN-Sprachberatung (Müller 1982, Scholze-Stubenrecht 1991, ders. 1995, ders. 2002), zum Aachener grammatischen Telefon (Stetter 1995, Mackowiak/ Steffen 1991), zum Essener Sprachtelefon (Cölfen 1996), zum Düsseldorfer Sprachservicetelefon (Höhne 1990, ders. 1991a), zur Sprachberatungsstelle der Martin-Luther-Universität in Halle-Wittenberg (Kühn/Almstädt 1997) und zum Sprachberatungsdienst der Gesellschaft für deutsche Sprache (Kolde 1976, ders. 1980) genannt. Aus der praktischen Arbeit der Sprachberatung resultieren auch die publizierten Sammlungen von Zweifelsfällen, die in der kommerziellen Lexikographie herausgegeben wurden (DUDEN Bd. 9, WAHRIG Bd. 5). Das Gegenstück dazu aus der ehemaligen DDR ist Dückert/Kempcke (1989). Zum Teil sprachtheoretisch interessiert, nicht praktisch-sprachberatend ist die Sammlung von Doppelformen in Muthmann (1994). Die angeführten Sammlungen sind prinzipiell auf die deutsche Gegenwartssprache gerichtet. In sprachhistorischer Perspektive gibt es zudem eine nicht zu vernachlässigende Zweifelsfall-Literatur, die spätestens im 18. Jahrhundert einsetzt und ihren Höhepunkt in den Jahrzehnten vor dem Ersten Weltkrieg besaß. Auch aus dieser Texttradition, die unten im zweiten Teil näher betrachtet wird, können Hinweise auf die Existenz von Zweifelsfällen abgeleitet werden (siehe unten Teil II). Der Vorteil der Datenbanken von Sprachberatungsstellen und der Zweifelsfallsammlungen liegt auch darin, dass hier mit Sicherheit von der Existenz metasprachlicher Reflexion ausgegangen werden kann. Die Konsultation von Sprachberatungsstellen und die Lektüre bzw. Erstellung von Zweifelsfallsammlungen ist nämlich nicht ohne eine metasprachliche Komponente denkbar.

Gegenüber den mittelbaren Zugängen zu den Zweifelsfällen in den Sprachberatungsdatenbanken und den Zweifelsfallsammlungen kann man versuchen in empirischen Erhebungen unmittelbar zu ermitteln, welche Zweifelsfälle zu einem gegebenen Zeitpunkt existieren $(=d$.). So sind beispielsweise Umfragen und experimentelle Sondierungen verschiedenster Art 
denkbar. Die Schwierigkeit dieser Methode liegt freilich - wie üblich - darin, dass die Art der Fragestellung gut bedacht sein muss. Denn es ist zu vermeiden, dass durch das Profil der Fragen gewisse Vorentscheidungen getroffen werden und die sprachliche Aufmerksamkeit der Probanden bereits in eine gewisse Richtung gelenkt wird. Bei der Untersuchung der Zweifelsfälle, die ohnehin für suggestive Thematisierungen anfällig sind, ist das beonders wichtig.

Diese Problematik kann in dem Versuch nicht auftauchen, sich unmittelbar an natürlichen metasprachlichen Thematisierungen von Zweifelsfällen zu orientieren $(=\mathrm{c}$.). Eine solche Methode muss gleichsam als Königsweg zu den Zweifelsfällen erscheinen, weil sie unvermittelt die Sprachwirklichkeit in den Blick nimmt und kein experimentelles setting produziert, das verzerrend wirken könnte. Der Nachteil dieser Methode liegt allerdings darin, dass es sehr schwer sein dürfte, so eine nennenswerte Zahl von Zweifelsfällen mit einem vertretbaren Aufwand zu identifizieren. Fraglich ist insbesondere, in welchen (mündlichen? schriftlichen?) Texten bzw. Textsorten man mit Aussicht auf Erfolg authentische Belege suchen dürfte. Sehr viel einfacher erscheint da die Befragung des eigenen Sprachgefühls, das ja als "linguistic intuition" auch in den gegenwärtigen sprachtheoretischen Fundierungen der Linguistik einen prominenten Platz einnimmt (= f.). Für diesen linguistischen Weg der Datensammlung gibt es durchaus auch in anderen Zusammenhängen gute Argumente (Gauger et al. 1982). Die Gefahr der Berufung auf das eigene Sprachgefühl besteht freilich darin, dass entsprechende Ansätze immer wieder drohen, in bloßen Subjektivismus abzugleiten: Was man selber qua Sprachgefühl für einen Zweifelsfall erklärt, muss längst nicht immer im gesamtsprachlichen Rahmen ein solcher sein.

Wiederum einen bloß vermittelten Zugang zu sprachlichen Zweifelsfällen gestattet der Weg über die bestehenden Wörterbücher und Grammatiken einer Sprache (=e.). Denn obwohl der Terminus Zweifelsfall kein eingebürgerter linguistischer Terminus ist, kommt er sachlich doch gelegentlich in lexikographischen und grammatischen Darstellungen einer Sprache zum Tragen. Ich möchte hier nur darauf aufmerksam machen, dass bei der Diskussion von primärsprachlichem Material neben dem Asterisk (*) als Kennzeichen für Ungrammatikalität gelegentlich auch das Fragezeichen (?) benutzt wird. Damit wird angezeigt, dass ein sprachliches Phänomen weder eindeutig als ungrammatisch noch als grammatisch klassifizierbar ist. Manche Forscher erweitern sogar das entsprechende Symbolinventar und benutzen beispielsweise drei Zwischenstufen: "(*)" für einen "grammatisch wahrscheinlich unkorrekten Ausdruck", "?" für einen "Ausdruck von fraglicher Korrektheit" sowie "(?)" für einen "Ausdruck von möglicherweise fraglicher Korrektheit" (Engel 1988: [8]). In den Wörterbüchern spielen oft Klammern eine vergleichbare Funktion (z.B. "gern (auch: gerne)"). Dadurch können relativ ökonomisch Doppelformen bzw. Varianten gebucht werden, die stets als erste Hinweise auf das Vorliegen von Zweifelsfällen deutbar sind. Durch derartige Symbolgebräuche wird also quasi empirische Identifikationsarbeit geleistet, insofern für bestimmte Wortpaare die Möglichkeit eröffnet wird, sie als Zweifelsfälle zu kategorisieren.

Was die Wege der Identifizierung von Zweifelsfällen betrifft, lässt sich festhalten, dass unterschiedliche Zugänge existieren, die alle ihre Vor- und Nachteile besitzen. In der Forschungspraxis dürften insbesondere die Datenbanken der Sprachberatungsstellen, die beste- 
henden Zweifelsfallsammlungen sowie die Erkundungen des (eigenen) Sprachgefühls erste Möglichkeiten bieten, sich einigermaßen verlässlich darüber zu informieren, welche Zweifelsfälle es in einer Sprache zu einem bestimmten Zeitpunkt gibt. In etwas strikterer linguistischer Fassung sollte freilich bedacht werden, dass allen Zugängen je spezifische Mängel anhaften. Daraus folgt: im günstigsten Fall sind verschiedene Methoden zu benutzen, um so Zweifelsfälle einigermaßen zweifelsfrei identifizieren zu können.

\subsection{Klassifikation}

Auf der Basis einer ersten Sichtung der Zweifelsfälle kann man eine vorläufige Klassifikation vornehmen, um so die Spannbreite des Phänomens zu erfassen und eine gewisse Orientierung zu ermöglichen. Im einzelnen lassen sich die - gemäß der oben diskutierten Definition und Methodologie identifizierten - Zweifelsfälle natürlich nach verschiedenen Kriterien gliedern und entsprechend unterschiedliche Typen zusammenfassen. Ich möchte hier nur eine Klassifikation nach der linguistischen Systemebene (A.) und nach den vermutlichen Entstehungsursachen (B.) kurz erörtern:

\begin{tabular}{|l|l|}
\hline $\begin{array}{l}\text { A. Klassifizierung nach der } \\
\text { Systemebene: }\end{array}$ & $\begin{array}{l}\text { B. Klassifizierung nach der Entstehungs- } \\
\text { ursache: } \\
\text { Zweifelsfälle entstehen durch die Existenz } \\
\text { von -: }\end{array}$ \\
\begin{tabular}{ll} 
a.) phonetischer Zf & a.) Sprachwandel \\
b.) orthographischer Zf & b.) Sprachkontakt \\
c.) morphologischer Zf & c.) Regiolekten/Dialekten \\
(Flexion, Wortbildung) & d.) Fachsprachen \\
d.) syntaktischer Zf & e.) Stillagen \\
e.) lexikalischer Zf & f.) Regelkonflikten im Sprachsystem \\
f.) semantischer Zf & g.) gesellschaftlich relevanten meta- \\
g.) pragmatischer Zf & sprachlichen Interventionen \\
\hline
\end{tabular}
\end{tabular}

Die Klassifizierung nach der Systemebene (A.) ist linguistisch einschlägig, deshalb allerdings nicht zu vernachlässigen. Was die Verteilung der Fälle nach diesem Kriterium angeht, so spricht alles dafür, dass orthographische Zweifelsfälle besonders zahlreich vorkommen (Höhne 1990, Mackowiak/Steffen 1991, quantitativ etwas abweichend Kolde 1976: 40f). Freilich ist damit zu rechnen, dass viele orthographische Zweifelsfälle mittelbar auch als syntaktische oder morphologische klassifiziert werden können. Phonetische Zweifelsfälle stehen dagegen vermutlich eher am unteren Ende der Frequenzskala. Die Verteilung könnte sich freilich ändern, wenn man bei einer Sprache neben dem indigenen Kernbereich systematisch auch eine regelrechte Fremdwortgrammatik ansetzt (am Beispiel des Deutschen Eisenberg 2001, ders. 2002). In derart geschiedenen Komponenten dürfte es zu unterschiedlichen Frequenzverteilungen von Zweifelsfällen in systemlinguistischer Sicht kommen. Phonetische Zweifelsfälle etwa sind in der Fremdwortgrammatik sicherlich häufiger als im nativen Kernbereich. 
Die Klassifizierung nach den Entstehungsursachen (B.) führt ins Herz der Zweifelsfallproblematik. Bevor die verschiedenen Typen kurz erläutert werden, soll vorab eine generelle Betrachtung zur Frage der Ursachen angestellt werden. Schon die Verankerung der Zweifelsfälle in metasprachlichen Sequenzen und in einem normativen Sprachbewusstsein verweist nämlich auf den allgemeinen Entstehungshorizont der Zweifelsfälle. Ohne ins reine Spekulieren abgleiten zu wollen, lässt sich wohl konstatieren, dass man es bei den Zweifelsfällen mit Konsequenzen von Schriftlichkeit zu tun hat. Erst im Kontext verschrifteter Sprachen, die ja generell wesentlich stärker standardisiert sind als unverschriftete, wird es verschärft zu Fällen sprachlichen Zweifels kommen. Darauf weist auch die Tatsache hin, dass Zweifelsfälle besonders häufig in Situationen auftauchen, in denen Schriftsprache produziert bzw. reflektiert wird (z.B. Höhne 1990, 1991a, Mackowiak/Steffen 1991). Wer einen Text schreibt, wird sicher wesentlich öfter von sprachlichem Zweifel geplagt sein als ein Sprecher, der lediglich alltagssprachlich vor sich hin parliert. Die unvermeidliche Aufmerksamkeit beim Schreiben und Lesen fördert ein verschärftes und ein spezifisches Bewusstsein von Sprache, durch das Zweifelsfälle sozusagen erst geschaffen werden. Die Schriftbezogenheit weist zudem - wie schon wiederholt angedeutet - darauf hin, dass für die Zweifelsfälle und das damit verbundene metasprachliche Bewusstsein die Phase des (schulischen, gesteuerten) Schriftspracherwerbs von besonderer Relevanz ist. Ferner steht zu vermuten, dass Zweifelsfälle besonders oft von denjenigen Sprecherkreisen thematisiert werden, die häufig und reflektiert Texte schreiben.

Auch die erläuterte Teilidentität der Varianten (s.o. Definition (d.)) bekommt erst im Zusammenhang schriftlicher Kommunikationskulturen eine besondere Brisanz. Denn das Strukturmuster der Schrift, in der formseitig keine Gradunterschiede möglich sind, sondern stets digitale Unterschiede gemacht werden müssen (z.B. entweder Kindes oder Kinds, mit oder ohne $e$, tertium non datur), liefert offensichtlich die Folie, vor der Zweifelsfälle, bewusst oder unbewusst, entstehen und bewältigt werden. Vor dem Horizont prototypischer Schreibprozesse der Gegenwart formuliert: entweder man drückt die (Buchstaben-) Taste einer ComputerTastatur oder man drückt sie nicht; technisch und daher auch kommunikativ ist es irrelevant, eine Taste nur ein bisschen oder etwa besonders heftig zu drücken. Selbst der konfliktäre Charakter vieler Diskussionen über Zweifelsfälle wurzelt vielleicht darin, dass konzeptuelle Folgelasten der Schriftlichkeit - entweder Variante a oder Variante b - zum Tragen kommen und ungerechtfertigterweise für die Sprache allgemein gelten sollen. Was in gesprochener Sprache (vor dem Hintergrund der Schriftsprache!) undeutlich, verwaschen und gegebenenfalls auch begrifflich unbewältigt bleiben kann, droht in den Produktions- und Rezeptionszusammenhängen schriftlicher (standardisierter) Sprache zu einem (systematischen) Anlass für Zweifel zu werden. Wie im Fall der Sicht auf die Lautsprache (Günther 1995), könnte man das sogar so weit treiben, in den symbolisch-konzeptuellen Vorgaben der Schriftlichkeit den generellen Hintergrund zu sehen, der die Wahrnehmung und Bewältigung der sprachlichen Zweifelsfälle konstitutiv bestimmt.

Im einzelnen können verschiedene Entstehungsursachen von Zweifelsfällen ausgemacht werden, die mehr oder weniger eng mit der allgemeinen Schriftbasiertheit zusammenhängen. Wie der obigen Tabelle zu entnehmen ist, resultiert die Existenz vieler Typen einerseits aus der Varietäten- bzw. Registervielfalt einer Sprache, andererseits aus dem Umstand, dass es sich 
bei Sprachen stets um gesellschaftlich verankerte, historisch wandelbare Gebilde handelt. So kann die Existenz von Fachsprachen, Regiolekten und Stillagen zu Zweifelsfällen führen, ebenso natürlich Sprachwandelprozesse und Sprachkontaktsituationen. Letzteres ist insbesondere bei den vielen Zweifelsfällen virulent, die mit der Fremdwortproblematik verbunden sind. Die gesellschaftliche Verankerung der Sprache führt auch immer wieder dazu, dass ambitionierte Sprechergruppen qua Sprachveränderung zur Gesellschaftsveränderung beitragen wollen. Dadurch werden mittelbar oft Zweifelsfälle geschaffen, wie etwa die feministische Sprachreflexion oder die Bemühungen um sog. politische Sprachkorrektheit zeigen. Im Zuge derartiger Bewegungen wurde es beispielsweise für viele Sprecher zweifelhaft, ob man nun im Deutschen Studenten oder Studierende zu sagen hat oder ob das Wort Neger überhaupt noch gängig ist (DUDEN Bd. 9:674-676). In vergleichbarer Hinsicht führen grundlegende gesellschaftlichspolitische Entwicklungen immer wieder zur Entstehung von Zweifelsfällen, so zuletzt etwa die deutsche Wiedervereinigung (Kühn 2003). Auch die sogenannten brisanten Wörter (Harras et al. 1989) und die Fahnenwörter (Stötzel/Eitz (eds.) 2002) stellen ein Reservoir für Zweifelsfälle dar.

Zuletzt ist allerdings diesseits der Varietätenvielfalt und der sozialen Dimension der Sprache deutlich darauf hinzuweisen, dass Zweifelsfälle auch direkt im Sprachsystem verankert sein können. Die Existenz etlicher Zweifelsfälle lässt sich gerade nicht auf die Registervielfalt zurückführen, auch wenn in verschiedenen Varietäten möglicherweise unterschiedliche Präferenzen herrschen mögen. Das gilt beispielsweise für grammatische Kongruenzprobleme, deren Analyse (zunächst) ganz ohne historische oder varietätengrammatische Aspekte erfolgen kann. Jaeger (1992) diskutiert eine Vielzahl derartiger Phänomene, die immer wieder zu besonderen Beschreibungs- und Kodifizierungsproblemen führten und vermutlich auch in $\mathrm{Zu}$ kunft führen werden (DUDEN Bd. 9: 515-540, Dückert/Kempcke 1989: 276-279; im Zusammenhang der Sprachberatung Siebert-Ott 1990). Auch die Probleme mit dem Konjunktiv können wahrscheinlich in diese Klasse eingeordnet werden.

\section{$2.4 \quad$ Klärung}

Zuletzt lassen sich die Zweifelsfälle in sprachpraktischer Perspektive nach den Klärungsmöglichkeiten klassifizieren. Dabei gehe ich davon aus, dass man hypothetisch von einer idealen Sprachberatungssituation ausgehen kann: Ein Sprecher artikuliert gegenüber einer Sprachberatungsstelle einen Zweifelsfall und bittet um einen Ratschlag zu dessen Klärung. Im folgenden Gespräch ist auf beiden Seiten genug Zeit und guter Wille vorhanden, sich etwas genauer mit den Varianten eines Zweifelsfalls zu befassen, um daraus letztendlich Gebrauchsempfehlungen abzuleiten. Bei der Auflösung des Zweifelsfalls bezieht sich die Sprachberatung auf den standardsprachlichen Usus, gegebenenfalls auch auf Gebräuche in relevanten Non-Standard-Varietäten. Das Spektrum des Usus wird so gut wie möglich deskriptiv geklärt, damit der jeweilige Sprachgebrauch als argumentative Basis für die Klärung fungieren kann.

Generell sind hier zwei Unterkriterien zu unterscheiden, die zur Lösung des Zweifelsfalls herangezogen werden können. Zunächst kann allein die Gebrauchsfrequenz der Varianten in der Standardsprache betrachtet werden (A.); weitere Überlegungen zum grammatischen Status 
oder dem Gebrauchskontext (B.) tauchen nicht auf. Aus einer solchen frequenzbasierten Erwägung ergeben sich (mindestens) drei Grundtypen: Die beiden Varianten sind ohne wesentliche Unterschiede gebräuchlich und insofern gleichermaßen richtig (freie Variation). Die eine Variante ist gebräuchlicher als die andere und insofern richtiger (graduelle Variation). Die eine Variante ist gebräuchlich, die andere ungebräuchlich (Null-Variation). Die frequenzbezogene Klärung berücksichtigt also den Umstand, dass standardsprachlich bestimmte Häufigkeitsverteilungen vorliegen können, die einen Zweifelsfall ohne Bezug auf weitere Analysehorizonte klären können und so gewisse Empfehlungen nahe legen.

Demgegenüber sind auch Klärungshorizonte denkbar, bei denen nicht aus der bloßen Frequenz, sondern aus dem Gebrauchskontext (im weitesten Sinne) Anhaltspunkte zur Lösung eines Zweifelsfalls ableitbar sind (B.). In dieser Perspektive können teilweise die Entstehungsursachen in den Blick geraten, insofern sich aus den varietätengrammatischen Hintergründen ebenfalls empfehlende Hinweise zum Gebrauch der Varianten ergeben. Entsprechend kann der Hinweis erfolgen, dass einzelne Varianten standardsprachlich ungebräuchlich, also unkorrekt, sind, während sie in regionalen, fachsprachlichen oder umgangsssprachlichen Varietäten durchaus gängig (korrekt) sein können (regionale -, fachsprachliche -, umgangssprachliche Variation). Eine vergleichbare Lösung liegt auch darin, dass manche Varianten unterschiedlichen Stil- oder Zeitschichten zugeordnet werden können (stilistische -, historische Variation). Denkbar ist zuletzt, dass allein der unmittelbare Sprach- bzw. Satzkontext, unahängig von allen kommunikativ-sozialen Hintergründen, ein (quasisystemlinguistisches) Kriterium dafür abgeben kann, wie ein gegebener Zweifelsfall zu klären ist. Daraus ergibt sich in der Regel eine komplementäre Variation, insofern die Varianten unterschiedliche Bedeutungs- bzw. Funktionswerte haben können. Die semantisch-syntaktische Analyse dieser Variation führt dann zu dem Ergebnis, dass im Sprachkontext a' stets Variante $a$, in b' stets b richtig ist. Hier handelt es sich also um eine (semantische) Disambiguierung von Varianten, die bereits auf der Basis des standardsprachlichen Usus erfolgen kann. Diese Analyse kann je nach Umstand der Beratung durch unterschiedlich tiefe grammatische Argumentation gestützt werden.

In der Übersicht stellt sich die Typologie der Klärungsmöglichkeiten demnach wie folgt dar: 
Zur Einführung in ein vergessenes Thema der Sprachwissenschaft.

\section{Klärungsmöglichkeiten}

(Bezugspunkt: mögliche Anfrage in einer Sprachberatungsstelle):

\section{Klassifikationskriterium: Frequenz (A.)}

1. Freie Variation: a und b ohne Restriktionen gebräuchlich (richtig)

(z.B. siebte/siebente, gern/gerne, Das Dorf wird der/in die Stadt eingegliedert.)

2. Graduelle Variation: a gebräuchlicher (richtiger) als b

(z.B. das/der Balg, magerer/magrer, dubios/dubiös, des Diesels/des Diesel)

3. Null-Variation: a ist gebräuchlich (richtig), b ist nicht gebräuchlich (falsch)

(z.B. Felsblöcke/Felsblocks, Er oder ich hat/habe das getan.)

\section{Klassifikationskriterium: Gebrauchskontext (B.)}

1. Stilistische Variation: a hat andere stilistische Konnotation als b

(z.B. baldmöglichst/möglichst bald, größer als/denn ich, jeder/jedweder)

2. Regionale Variation: a standardsprachlich, b regiolektal

(z.B. Erlasse/Erlässe, die Ersparnis/das Ersparnis, Ochse/Ochs)

3. Fachsprachliche Variation: a standardsprachlich, b fachsprachlich

(z.B. Mütter/Muttern, Klage über/gegen, die Niete/der Niet)

4. Umgangssprachliche Variation: a standardsprachlich, b umgangssprachlich

(z.B. für das/fürs, Jungen/Jungens/Jungs (Pl.), herumlaufen/rumlaufen)

5. Historische Variation: a heute, b früher gebräuchlich (richtig)

(z.B. Likör/Liqueur, Franzose/Franzman, Frömmigkeit/Frommheit)

6. Komplementäre Variation: im Sprachkontext $\mathrm{x}$ ist a richtig, im Sprachkontext y ist b richtig (z.B. hat/ist geflattert, der/das Moment, jährlich/jährig, lösbar/löslich).

\subsection{Einige weitere Schlussfolgerungen}

Aus der skizzenhaften Typologie der Zweifelsfälle, wie sie in den vorangegangenen Abschnitten formuliert wurde, lassen sich einige weitere Konsequenzen ziehen. Zunächst zeigen die Betrachtungen, dass die Zweifelsfälle sehr heterogen sind. Ihr sprachliches Profil ist kaum auf einen einheitlichen, einfachen Nenner zu bringen: Sie sind systemlinguistisch nicht auf eine Sprachebene festgelegt, ihre Entstehungsursachen sind vielgestaltig, entsprechend ihre Klärungsmöglichkeiten. Insgesamt handelt es sich also um ein recht komplexes Phänomen. Daraus ist vor allem der Schluss zu ziehen, dass man mit generalisierenden Aussagen über "die Zweifelsfälle" sehr vorsichtig sein sollte. Denkt man sich die Zweifelsfälle als Gegenstand der Linguistik, so wird es sicher sinnvoll sein, jeweils spezifische Fälle nach bestimmten Kriterien und mit bestimmten Zielen herauszugreifen.

Die Vielgestaltigkeit der Zweifelsfälle lässt sich auch angesichts der obigen Überlegungen interpretieren, die die kompetenztheoretische Einbindung der Zweifelsfälle problematisierten. Ganz allgemein kann man nämlich annehmen, dass das Bewusstsein von Zweifelsfällen in dem Maße zunimmt, als die Sprecher der Varietätenvielfalt und der komplexen Struktur einer Sprache gewahr werden. Anders formuliert: je mehr ein Sprecher von den Verwinkelungen und vielschichtigen Formulierungsmöglichkeiten einer Sprache weiß, desto eher wird er bei Gelegenheit auch von Gefühlen der Unsicherheit und des Zweifels geplagt werden. In dieser 
Linie sind die Zweifelsfälle also kein Zeichen für sprachliche Inkompetenz, sondern gerade von Kompetenz. In einem vergleichbaren Sinn wurde etwa auch von der "kompetente[n] Unsicherheit" (Mackowiak/Steffen 1991:534) gesprochen, die sich in der Tatsache ausdrückt, dass oft besonders sprachversierte Sprecher Sprachberatungsstellen konsultieren.

Sehr zugespitzt könnte man vielleicht sogar davon ausgehen, dass viele Sprachzweifel - zumindest zum Teil - auf eine hohe Sprachkompetenz, besser vielleicht: Sprachsensibilität verweisen, insofern die Zweifelnden Lücken, Dissonanzen und Unstimmigkeiten einer komplex strukturierten Sprache wahrnehmen. Damit besitzt man sozusagen ein realistischeres Bild einer Sprache und ihres eigentümlichen ontologischen Status, als wenn man stets in subjektiver Gewissheit überhaupt keine Varianten zur Kenntnis nimmt oder zwischen ihnen stets problemlos entscheidet. Im mehr oder weniger reflektierten Bewusstsein der Zweifelsfälle drückt sich so nicht zuletzt die Tatsache aus, dass Legitimation in sprachlichen Dingen nicht in der individuellen Überzeugung, sondern in der komplex strukturierten Gemeinschaftlichkeit einer Sprache liegt, die paradoxerweise nur in individuellen sprachlichen Akten greifbar ist.

Eine andere Frage ist es natürlich, ob die Zweifelnden selber ihren Zustand als spezifische Form der Kompetenz begreifen. Wahrscheinlich wird eher das Gegenteil der Fall sein: Wer zweifelt, wird sich vielmehr als sprachinkompetent, gar als ungebildet empfinden. Diese Reaktion ist aber nicht naturwüchsig, sondern gewiss durch den gesellschaftlichen, insbesondere den schulischen, Umgang mit sprachlichen Zweifelsfällen geprägt. An dieser Stelle bietet sich infolgedessen ein Übergang zur metasprachlichen, soziolinguistischen Problematik an, wie und mit welchen Konsequenzen die Zweifelsfälle denn in einer Sprachgemeinschaft behandelt werden und wurden.

\section{Zur metasprachlichen Geschichte und Gegenwart der Zweifelsfälle im Deutschen}

Wie im anfänglichen Teil gezeigt, sind sprachliche Zweifelsfälle mit metasprachlichen Gehalten verbunden. In der zweifelnden Reflexion über Varianten liegt immer eine Form von bewusster Überlegung. Auch wenn diese Feststellung auf den individuellen Akt des Zweifelns zielte, so lässt sich doch der Frage nachgehen, ob und, wenn ja, auf welche Art und Weise Zweifelsfälle im allgemeinen Rahmen einer Sprachgemeinschaft thematisiert werden. Denn was viele Sprecher immer wieder zum Zweifeln bringt, wird wahrscheinlich auch zu einschlägigen gesellschaftlichen Institutionen und Strategien der Problembewältigung führen. Es ist nicht unwahrscheinlich, dass beim gesellschaftlichen Umgang mit Zweifelsfällen auch je spezifische (national-) sprachliche Traditionen wirken. In diesem Sinne möchte ich mich im Teil II exemplarisch mit der deutschsprachigen Tradition der Reflexion über Zweifelsfälle beschäftigen. Selbstverständlich ist im Rahmen dieses Aufsatzes keine detaillierte Beschäftigung mit der einschlägigen Überlieferung möglich. Das verhindert schon allein der reine Umfang der einschlägigen Schriften. Es handelt sich um ein umfangreiches, inhaltlich schillerndes Text-Korpus. ${ }^{8}$ Es soll aber zumindest in Ansätzen und einigen großen Linien gezeigt werden, dass und wie bei der Beschäftigung mit Zweifelsfällen immer auch gewisse meta-

\footnotetext{
${ }^{8}$ Vgl. die Quellenliste am Ende, die sicher noch nicht vollständig ist; zum sprachgeschichtlichen Kontext generell Cherubim 1983.
} 
sprachliche Traditionen und Bewegungen berücksichtigt werden können, ja müssen. Wenn man sich sprachwissenschaftlich mit Zweifelsfällen befasst, genügt es also nicht, objektsprachliche Daten in system- und varietätenlinguistischer Perspektive zur Kenntnis zu nehmen. Denn in vielen Fällen sind die Zweifelsfälle zugleich Bestandteile in einer teilweise weit zurückreichenden Geschichte der (öffentlichen) Sprachthematisierung und Sprachdiskussion. Die Relevanz dieser Kontexte spricht sprachbewusstseinsgeschichtlich und soziolinguistisch zunächst einmal für sich. Ferner sind aber auch die konkreten Auswirkungen dieser Diskussion auf die objektsprachliche Entwicklung des Deutschen bis heute in vielen Details noch völlig ungeklärt. In den geläufigen Theorien zum Sprachwandel, die meistens auf relativ dünnen empirischen Fundamenten gebaut sind, werden sie jedenfalls kaum wirklich thematisiert (z.B. Keller 1994, tendenziell anders aber Neuland 1993).

Es ist nicht ganz klar, wer warum zum ersten Mal den Begriff des sprachlichen Zweifelsfalls prägte oder sachlich nur in seine Nähe geriet. Einiges deutet freilich darauf hin, dass der Einsatzpunkt entsprechender Überlegungen irgendwo am Beginn des 18. Jahrhunderts liegen muss. Dafür möchte ich hier nur zwei Belege anführen. Zunächst war es Leibniz, der in seiner Schrift zum Deutschen von sprachlichen "Zweifelsknoten" sprach, die unter der Maßgabe von "Sprachrichtigkeit" "aufzulösen" seien. Im Blick auf die jüngsten Umbrüche der damaligen Entwicklung des Deutschen kam er darauf zu sprechen, dass auf sprachinteressierter Seite sozusagen Handlungsbedarf bestand:

denn obwohl darin [d.i. auf dem Feld der "Sprachrichtigkeit" (WPK)] ziemlicher Mangel befunden wird, so ist es doch nicht schwer, solchen mit der Zeit zu ersetzen und sonderlich vermittelst guter Überlegung zusammengesetzter tüchtiger Personen einen und anderen Zweifelsknoten aufzulösen.

(Leibniz 1717: §102))

Einige Jahrzehnte später veröffentlichte der süddeutsche Gelehrte Ignaz Weitenauer ein Buch, in dem der Begriff des sprachlichen Zweifels und seiner Lösung bereits im Titel stand: Zweifel von der deutschen Sprache vorgetragen, aufgelöset, oder andern aufzulösen überlassen (Weitenauer 1764). ${ }^{9}$ Obwohl es bei den Erörterungen von Leibniz und Weitenauer sicher einige konzeptionelle Unterschiede gibt, so existiert doch ein genereller Formulierungshorizont, der auch für die weitere Beschäftigung mit den Zweifelsfälle maßgeblich werden wird. Sowohl Leibniz als auch Weitenauer siedelten ihre Stellungnahmen im Umkreis eines großen sprachbewussten Projekts an. Beide befassten sich nämlich mit der Tatsache, dass in sprachlichen Dingen bei vielen Menschen ein Orientierungsbedarf bestand. In mannigfaltigen Einzelheiten war noch unklar, wie aus der Vielfalt der deutschen Sprechweisen, namentlich: der vielen dialektalen Varianten, eine einheitliche deutsche Standardsprache mit hohem Prestige und alle Varietäten übergreifend entstehen könnte. Anders gesagt: die beiden Gelehrten wollten durch die Auflösung sprachlicher Zweifelsfälle dazu beitragen, dass die allseits für positiv erachtete Vereinheitlichung und Kultivierung der deutschen Sprache vorangetrieben wurde. Angesichts des Umstands, dass der tatsächliche Sprachgebrauch in diesem Punkt keine Legi-

\footnotetext{
${ }^{9}$ Zum philologisch-linguistischen Wirken Weitenauers und seinem biographischen Profil vgl. Jahreiß 1990: 96-
} 104. 
timation bieten konnte - schließlich beriefen sich die Sprecher aller Regionen auf den je eigenen Gebrauch - bevorzugten beide eine Rechtfertigungsinstanz, die unmittelbar dem aufklärerischen Geist der Zeit entstammte. Leibniz wollte die "gute Überlegung" "tüchtiger Personen" befragen; auf derselben Linie formulierte Weitenauer den Vorrang der "Vernunft" und der vielleicht cartesianisch inspirierten - "gründlichen Gewißheit" vor den einzelnen landschaftlichen Sprachgebräuchen:

Kein Land wird jemals das andre für seinen Schiedsrichter erkennen, (...) Aber könnte nicht etwann die Vernunft selbst in vielen Stücken einen Ausspruch thun, und in andern einen ehrlichen Vergleich vorschlagen? (...) Werde ich je zuweilen das Glück haben, auf eine gründliche Gewißheit zu kommen: so bin ich eben darum befugt den vorgetragenen Zweifel aufzulösen und zu entscheiden.

(Weitenauer 1764:Vorrede)

Ich möchte nicht behaupten, dass Leibniz und Weitenauer in allen späteren Diskussionen die Leitfiguren bei der Behandlung der Zweifelsfälle abgaben. Es kommt mir hier vielmehr darauf an, die grundsätzliche Konstellation zu beleuchten, die im 18. und auch noch in weiten Teilen des 19. Jahrhunderts die Wahrnehmung der Zweifelsfälle dominierte. Als Zweifelsfall erschienen vor allem diejenigen Momente der Sprachwirklichkeit, die der Vereinheitlichung des Deutschen noch entgegenstanden. Wer zweifelt, ist - so die damalige Überzeugung - noch zu sehr an die Schranken seiner landschaftlichen Herkunft und die Wankelmütigkeiten seines bloß individuellen Sprachgefühls gebunden. Ihm fehlt die gesellschaftliche Verankerung in einem übergreifenden kommunikativen Standard. Man sah die jüngst sehr positiv verlaufene deutsche Sprachentwicklung noch nicht auf dem Gipfel, da in vielen Details noch Zweifel bestanden, wie der Standard aussehen sollte.

Einen Ausweg aus dieser Situation erhoffte man sich in der vernunftorientierten Reflexion und einer entsprechenden Erziehung, durch die jeder sprachliche Zweifelsfall aufgelöst und somit die heterogene sprachliche Verfassung überwunden werden sollte. Die Lösung der Zweifelsfälle ist vor diesem Hintergrund ein kollektives Unternehmen, das die sprachliche Situation in Deutschland im Dienste der Einheitlichkeit befördern sollte. Im Hintergrund wirkte dabei die verbreitete Überzeugung, dass Varianten und Doppelformen sozusagen Defekte darstellen, die in einer gesellschaftlichen Anstrengung behoben werden könnten, ja müssten. ${ }^{10}$ Mindestens untergründig, oft aber auch ausdrücklich bestanden Beziehungen der Literatur, die sich mit Zweifelsfällen befasste, zu den traditionell grammatisch-lexikographischen Beschreibungen des Deutschen (v. Polenz 1994: Kap. 5.5.-5.7).

Im skizzierten Unternehmen war nun nicht immer ausdrücklich von Zweifelsfällen die Rede. Ganz generell konnte es beispielsweise um "Anweisungen" gehen, das Deutsche angemessen und korrekt zu gebrauchen (Heynatz 1785, Angerstein 1791, Schmiedtgen 1799). Auch die ausdrückliche Orientierung an der "Sprachrichtigkeit" bezeugt, dass in entsprechenden Publi-

\footnotetext{
${ }^{10}$ So stellte z.B. Henrich Bauer fest, dass Doppelformen "allen Sprachlehrern zuwider sind, und daß man sich viel Mühe giebt, sie auf verschiedene Art wenigstens der Form nach aus der Grammatik zu entfernen" (Heinrich Bauer, Vollständige Grammatik der neuhochdeutschen Sprache, II, Berlin 1828 S. 226; zit. nach Joeres 1996: 304).
} 
kationen praktisch dieselbe Ambition verfolgt wurde (Stutz 1789). Puristische Horizonte konnten hier sinnvoll eingegliedert werden (Heynatz 1796). Nach dem Selbstverständnis der Autoren ging es stets um die "Verbesserung" des Deutschen (Heynatz 1801). Auch die um die Wende zum 19. Jahrhundert einsetzenden populären Bewegungen, die sich mit Problemen der Sprache auseinandersetzten, griffen in dieser Art und Weise auf die Zweifelsfälle zu. So bekannte beispielsweise Heinsius, dass die Klärung von Zweifelsfällen einer der Gründe gewesen sei, warum er seinen Sprach- und Sittenanzeiger gegründet hatte. ${ }^{11}$

Im Laufe des 19. Jahrhunderts kam es nun zu einigen bezeichnenden Entwicklungen im Rahmen der Beschäftigung mit den Zweifelsfällen. Zunächst erschienen resümierende Sammlungen, in denen das mittlerweile angehäufte Material nach der Art von Nachschlagewerken zusammengetragen wurde (Ditscheiner 1847). Daniel Sanders verfasste mit großer Resonanz eine alphabetisch geordnete Zusammenstellung von Zweifelsfällen (Sanders 1872). Sein Buch avancierte schnell zu einem weitverbreiteten Bestseller, der 1908 bereits in der 44. Auflage herauskam. Generell orientierte sich Sanders bei der Behandlung der Zweifelsfälle weiterhin am Prinzip der Rationalität; dabei geriet der aktuelle Sprachgebrauch keineswegs aus dem Blick (vgl. auch Haß-Zumkehr 1995: 195-236). ${ }^{12}$ Unabhängig von den behandelten Details war die grundsätzliche Linie bei Sanders immer noch identisch mit den Ausblicken von Leibniz und Weitenauer: In einer nüchternen, rationalen Anstrengung sollten die Zweifelsfälle getilgt werden, damit die sprachliche Homogenisierung und Kultivierung Deutschlands vorangetrieben wurde.

Wie bei anderen Autoren seiner Zeit (Lühr 1992), wurde das Rationalitätsprinzip in sprachlichen Dingen insbesondere in der Orientierung an Redundanzvermeidung, Eindeutigkeit und Deutlichkeit ausformuliert. ${ }^{13}$ Dahinter stand meistens auch die Überzeugung, dass es eine unmittelbare Beziehung zwischen Sprache, Denken und Wirklichkeit gibt bzw. geben sollte (Dieckmann 1991: 364-369). Wo der uneinheitliche Sprachgebrauch keine rechte Orientie-

11 Als zukünftiges Arbeitsprogramm für seine Zeitschrift formulierte er folgenden Punkt: "Kurze Untersuchungen und Belehrungen über schwankende und streitige Formen (...), deren es in der deutschen Sprache (wie in allen nicht abgeschlossenen) viele giebt, und alle dahin gehörigen Anfragen beantworten." (Heinsius 1817:2) [Ich danke Manuela Böhm (Potsdam) für den Hinweis auf diese Quelle.]

12 Wegen der großen Bedeutung des Werks sei hier die schöne Stelle zitiert, in der der Leser eingangs mit der Brisanz der Zweifelsfälle vertraut gemacht wurde: "Es gibt im Deutschen, wie in jeder noch in lebendiger Fortentwicklung begriffenen Sprache, unberührt von den allgemein anerkannten Regeln, die allen Gebildeten geläufig und vertraut sind und gegen die sie deshalb niemals verstoßen werden, eine nicht geringe Anzahl von Fällen, in denen sich der Sprachgebrauch noch nicht - oder doch mindestens noch nicht ganz entschieden und zweifellos - festgestellt hat und in denen das Schwanken bei Gebildeten und selbst bei Schriftstellern eine Unsicherheit erzeugt, ob die in einem bestimmten Fall nebeneinander vorkommenden verschiedenen Formen und Ausdrucksweisen gleichberechtigt sind oder welche die richtigere oder vielleicht die allein richtige sein dürfte. Die Zweifelsfälle sind nicht bloß zahlreicher, sondern es ist auch die Unsicherheit in denselben größer, als man im allgemeinen glaubt und anerkennt." (Sanders 1872: Vorwort).

13 Z.B. Andresen 1923: 4f, dagegen streitet später Engel 1929: 21ff. Die Reflexionen über die angeblich besonders durch Logik geprägte deutsche Sprachentwicklung, die Karl Ferdinand Becker unter anderem in seinem Buch Der deutsche Stil (1848) vorgetragen hatte, mögen hier eine katalytische Wirkung gehabt haben (Dieckmann 1989: Kap. 21). 
rung zu geben versprach, galt also beispielsweise folgendes: Wenn eine logisch-gedankliche Unterscheidung gemacht werden kann, so soll sie sich auch eindeutig in bestimmten sprachlichen Formen ausdrücken. Damit verfügte man über ein Entscheidungskriterium, durch das sich nicht wenige Zweifelfälle entscheiden ließen. Unberücksichtigt blieb in einer solchen logikbasierten Klärung freilich, ob Sprecher in einer gegebenen Situation die jeweilige gedankliche Unterscheidung überhaupt machen wollten oder, verschärft gesagt, ob sie im jeweiligen Kommunikationskontext überhaupt fraglich war und insofern eindeutig markiert sein musste. Wie dem auch sei, methodologisch stand Sanders immer noch eindeutig auf dem Boden der Aufklärung und favorisierte überall dort das Rationalitätsprinzip, wo der Bezug auf den wankelmütigen Sprachgebrauch nicht weiterführte.

Gegenüber den Autoren des 18. Jahrhunderts hatte sich jedoch auch etwas nicht Unwesentliches geändert. Denn sein Werk war sowohl quantitativ als auch qualitativ viel ausgefeilter und umfangreicher als die Schriften der Vorgänger. Auf 450 eng bedruckten Seiten wurden die Zweifelsfälle in einer bisher ungekannten Detailfreudigkeit und Materialfülle, basierend auf primären Sprachdaten unterschiedlichster Art, thematisiert. Negativ formuliert könnte man freilich auch sagen, dass Sanders eine unglaublich unübersichtliche Sammlung sophistischer Haarspaltereien vorgelegt hatte und so das pedantisch-normative Raisonnieren über Sprache nachhaltig inspirierte. In dieser Sicht ließe sich sein Wörterbuch sogar als Bankrotterklärung interpretieren: Denn anders als die Autoren des 18. Jahrhunderts noch gehofft hatten, waren die Zweifelsfälle im 19. Jahrhundert ja nicht aus der Welt geschafft. Im Gegenteil! Durch die diversen Publikationen zu den Sprachschwankungen und Doppelformen, die nun im Werk von Sanders gipfelten, waren mit der Zeit immer mehr Zweifelsfälle bekannt und zum Problem geworden. Man könnte geradezu zu der These verleitet werden, dass die sprachbewusste Literatur die Zweifelsfälle nicht wie erhofft zum Verschwinden gebracht, sondern das Entstehen weiterer Problemfälle verursacht hatte. Geistesgeschichtlich gedeutet: die optimistischen, vernunftgeleiteten Ausblicke der Aufklärung hatten nicht das geleistet, was man sich von ihnen versprochen hatte.

In dieser Situation änderte sich der Tonfall und die Blickrichtung der Autoren, die sich mit Zweifelsfällen befassten. Die Existenz der Zweifelsfälle wurde nicht mehr nüchtern als Anstoß für ein rationales gesellschaftliches Projekt begriffen, sondern als Zeichen für Kulturverlust und eine allgemeine verderbliche Nachlässigkeit. Mit kulturpessimistischem und antimodernem Ressentiment sah man in sprachlicher Heterogenität jetzt "Sünden der Gegenwart" (Lehmann 1877); die Zeichen der Zeit sollten die "Verrottung" der Sprache anzeigen, die von berufenen Persönlichkeiten unbedingt "gerettet" werden müsste (v. Wolzogen 1880). Ausgangspunkt war also die mutmaßliche Beobachtung, dass die "Unsicherheit und Willkür der Sprachgestaltung immer schlimmer wird" (Matthias 1892: Vorwort) und von daher gegenwärtig "Sprachverderbnis" zu konstatieren sei (Briegleb 1911). Erst jetzt wurde der Topos des Sprachverfalls zu einem geläufigen Bild, das seitdem immer wieder weite Teile des öffentlichen Sprachbewusstseins dominiert (Klein 1986, Schrodt 1995, unscharf Beck 1996). Die zentrifugalen Kräfte der Sprachentwicklung, die früher in optimistischen Ausblicken bewältigt werden sollten, schienen nach der Ansicht dieser Autoren nun langsam den Verfall des Deutschen nach sich zu ziehen. 
Mit dieser Blickverschiebung war es auch verbunden, dass man sich nun nicht mehr vordringlich am Orientierungsbedürfnis der Sprachgemeinschaft in Zweifelsfällen orientierte. Vielmehr wurden jetzt angebliche Fehler und Nachlässigkeiten im Sprachgebrauch der Anderen identifiziert, ohne dass diese nach Rat oder Belehrung in sprachlichen Dingen gefragt hätten. Zugespitzt formuliert: statt nüchterner Sprachberatung aufgrund von Anfragen kam es zu erregter Kritik an der Sprache der Anderen. Sie wurde im schlimmsten Fall ganz und gar willkürlich, arrogant und denunziatorisch vorgetragen. Die Verschiebung von der adressatenbezogenen Sprachberatung zum unerbetenen Sprachtadel resultierte wahrscheinlich auch aus der zunehmenden Kluft zwischen der öffentlichen Sprachdiskussion und der wissenschaftlichen Sprachforschung, die sich mit historischen Forschungsschwerpunkten mittlerweile akademisch etabliert hatte. ${ }^{14}$ Sozialgeschichtlich lässt sich dieser Wandel überdies als eine Sprachkritik begreifen, die im Dienste sozialer Distinktion stand. Die meisten Sprachkritiker, die in der zweiten Hälfte des 19. Jahrhunderts die Stimme erhoben, taten dies, um sich als Bildungsbürger im Medium eines bestimmten Sprachgebrauchs ihres gesellschaftlichen Rangs zu versichern (Dieckmann 1991). Aus Zweifelsfällen und Sprachschwierigkeiten werden nun also unbewältigte Fehler und Sprachsünden, die sozialen Stigmata gleichkommen.

Das Paradebeispiel für die veränderte Haltung gegenüber den Zweifelsfällen ist sicherlich das einschlägige Werk von Gustav Wustmann: "Allerhand Sprachdummheiten. Kleine deutsche Grammatik des Zweifelhaften, des Falschen und des Häßlichen" (Wustmann 1892, dazu Henne 1966). Das derb formulierte Werk avancierte schnell zu einem erfolgreichen, viel gelesenen Vademecum sprachbewusster Kreise. Ausdrücklich wurde darin festgestellt, dass nun nicht mehr Vernunft und Sprachgebrauch die entscheidenden Legitimationsinstanzen abgaben, sondern ein stark dezisionistisch geprägtes ästhetisches Gefühl: der "gute Geschmack" (Wustmann 1903: Vorwort zur dritten Auflage XIII). Wustmanns Buch zog rasch Gegenschriften nach sich. Man wandte sich aber meistens nicht grundsätzlich gegen seine Intentionen, sondern missbilligte etwa lediglich den allzu scharfen Ton oder schlug in Einzelpunkten andere Lösungen vor. Faktisch blieb man insgesamt im sprachtheoretischen Gesichtskreis vom drohenden Untergang der Sprache befangen, obwohl in sprachlichen Details zwischen den Autoren durchaus gestritten wurde (Minor 1892, Kaerger 1892, Brunner 1895: bes. Kap. X, Contze 1898, Schmeitzner 1909). Ruhigere und analytischer angelegte Stimmen verloren gegenüber Wustmann und seinen Anhängern an öffentlicher Resonanz (Matthias 1892). Versuche, aus der einschlägigen Diskussion Konsequenzen für das Verhältnis von Sprachwissenschaft und öffentlicher Sprachdiskussion zu ziehen (Tappolet 1898), blieben praktisch folgenlos. Im Zuge der erhitzten sprachkritischen Debatte kam es vielmehr zu Spezialschriften, in denen die Zweifelsfälle in bestimmten funktionalen Varietäten thematisiert wurden. Zum einen wurde besonders die Fachsprache der Wirtschaft aufgearbeitet (Bennewitz 1898). Zum

\footnotetext{
${ }^{14}$ Zur Vorgeschichte, in der öffentliche Sprachdiskussion und linguistische Arbeit wesentlich weniger separiert waren, vgl. überzeugend Donhauser 1986, problematisierend Bergmann 1982, orientiert an grammatischen Details Habermann 1997, Jakob 1999, Konopka 1996. Für den Rückzug der Sprachwissenschaft aus der öffentlichen Diskussion ist im germanistischen Kontext vor allem Jacob Grimm und seine romantische Sprachtheorie verantwortlich zu machen.
} 
anderen gerieten die Zweifelsfälle mehr und mehr ins Visier der Pädagogen, die für den muttersprachlichen Deutschunterricht nach sinnvollen Unterrichtsgegenständen suchten (Matthias 1896, ders. 1897, Poetzsch 1897, Franke 1903, Fischer 1903).

Insgesamt lässt sich resümieren, dass die Zweifelsfall-Literatur in den drei Jahrzehnten vor dem Ersten Weltkrieg einen Höhepunkt erreichte. Es gab zwar auch später noch ähnlich angelegte Werke, die sich jedoch teilweise gegen die herkömmlichen Prinzipien der Sprachbewertung wandten. Engel (ausführlich Sauter 2000) etwa bezog gegen die alten "Sprachdrillmeister" und ihre Methoden Stellung; dabei billigte er auch ausdrücklich die Existenz von Doppelformen. ${ }^{15}$ Mit den Werken von Sanders, Wustmann, Andresen und Matthias lagen jedoch die maßgeblichen Werke vor, die sowohl nach der Sache als auch nach dem Tonfall die Marksteine der Diskussion von Zweifelsfällen im deutschen Sprachkontext gesetzt hatten. Ob freilich die erwähnten sprachkritischen Autoren tatsächlich das grundsätzliche Verständnis der deutschen Sprachgemeinschaft in puncto Wortbildung geprägt haben (Heringer 1984, Donalies 2003), möchte ich an dieser Stelle lieber offen lassen. Etwas tiefer gehängt wird man aber zumindest die Hypothese aufstellen können, dass noch die heutigen Stellungnahmen zu den Zweifelsfällen in der einen oder anderen Weise auf Motive und Gehalte der Sprachkritik des 19. Jahrhunderts zurückgeführt werden können. In den zeitgenössischen rhetorischstilistischen Ratgebern finden sich beispielsweise immer wieder Abschnitte, die an die älteren Texte erinnern (Bremerich-Vos 1991: 121ff, 140ff, Nickisch 1975, Püschel 1991).

Es ist Aufgabe der künftigen Forschung, genauer zu klären, welche Wirkungen die Zweifelsfall-Literatur in der deutschen Sprachgeschichte bis heute nach sich zog. Dabei wird zu unterscheiden sein, ob es um objekt- oder metasprachliche Fakten geht. Objektsprachlich könnte die Literatur an einigen Punkten tatsächlich sprachvereinheitlichend gewirkt haben, indem bestimmte Varianten, aus welchen Gründen auch immer, abgewertet wurden und ihr $\mathrm{Ge}$ brauch infolgedessen soziale Missbilligung hervorrief. In vielen Fällen wird sie aber auch ohne große Wirkungen auf den Sprachgebrauch geblieben sein (so, die wenig ausgebaute Forschung resümierend, v. Polenz 1999: 299f).

Metasprachlich wäre zu prüfen, ob bestimmte Haltungen zur Sprache generell von dem skizzierten Schrifttum gestützt oder sogar geschaffen wurden. Insbesondere scheint es mir interessant zu sein, möglichen Konsequenzen im Schulunterricht und dem dort anhand der Zweifelsfälle vermittelten Sprachbewusstsein nachzuspüren. Auch die Bewertung einzelner sprachlicher Phänomene könnte womöglich in vielen Punkten auf die - zumindest untergründige Wirkung der älteren Zweifelsfall-Literatur zurückgeführt werden. In jedem Fall ist eine Warnung zu berücksichtigen, die oben schon im systematischen Teil ausgesprochen wurde: Angesichts der Vielgestaltigkeit der Zweifelsfälle wird man sich vor allzu generalisierenden Aussagen hüten müssen. Erfolgversprechend dürften dagegen Perspektiven sein, in denen einzel-

\footnotetext{
15 "Doppelformen schaden keiner Sprache, der deutschen Sprache sind sie durch Anlage und Entwicklung ureigen." (Engel 1929:27). In diesem Sinne verteidigte Engel auch die "Sprachfreiheit" als grundlegendes "Gesetz der deutschen Sprache" (ebd.); diese Freiheit sei keineswegs ein Zeichen für Zügellosigkeit oder Schludrigkeit (28).
} 
ne Typen von Zweifelsfällen identifiziert und ihre objekt- und metasprachliche Geschichte und Gegenwart genauer als bisher erforscht wird.

\section{Schluss: Thesen zur Behandlung der Zweifelsfälle}

Der Aufsatz sollte mit sehr groben Strichen ein weites Feld abstecken. Dieser Ambition entsprechend, möchte ich mit resümierenden Thesen enden. Sie sollen noch einmal zusammenfassen und akzentuieren, dass und wie die Zweifelsfälle im Gegensatz zur bisherigen Praxis ein genuin linguistischer Gegenstand sein können, ja müssen. Nachzutragen ist hier zudem, was aus den obigen Ausführungen implizit schon hervorgegangen ist. Wer Geschichte und Gegenwart der Zweifelsfälle erforscht, sollte am besten mehrere Zugänge im Auge behalten. Einer systemlinguistisch-grammatischen Klärung sind mindestens sprachgeschichtliche und soziolinguistische Perspektiven an die Seite zu stellen; dazu können je nach den vorliegenden Befunden sprachbewußtseins- und wissenschaftsgeschichtliche Aspekte kommen. Bei den Zweifelsfällen handelt es sich also um einen Gegenstand, der sich hervorragend dazu eignet, die verschiedenen Ansätze und Schulen der Sprachwissenschaft ins Gespräch zu bringen, um so einen realistischen, ebenso strukturell-systemlinguistischen wie gesellschaftlich vermittelten Sprachbegriff in den Mittelpunkt der disziplinären Selbstvergewisserung zu stellen.

1. Der Begriff Zweifelsfall ist aus systematischen und historischen Gründen ein wichtiger sprachwissenschaftlicher Terminus. Er spielt sowohl objekt- als auch metasprachlich, sowohl systemlinguistisch als auch soziopragmatisch eine Rolle bei der Analyse natürlicher Sprache. In sprachwissenschaftlichen Wörterbüchern muss der Begriff Zweifelsfall als Terminus aufgenommen werden.

2. In der Existenz von Zweifelsfällen verkörpert sich die Tatsache, dass in einer Sprachgemeinschaft ein Orientierungsbedürfnis in sprachlichen Dingen besteht. Es gehört zu den Aufgaben der Sprachwissenschaft, sich mit diesem Orientierungsbedürfnis auseinanderzusetzen, indem sie auf deskriptiver Basis normativ interpretierbare Anhaltspunkte zur Klärung der Zweifelsfälle identifiziert.

3. Die empirische Identifikation der Zweifelsfälle ist strikt von deren möglicher Klärung $\mathrm{zu}$ unterscheiden. Sowohl in der Identifikations- als auch in der Klärungsperspektive besteht Forschungsbedarf:

a. Was sind in der Gegenwartssprache tatsächlich Zweifelsfälle (Identifikation)?

b. Welche empirischen Ergebnisse und theoretischen Konzeptualisierungen ergeben sich aus näheren Untersuchungen zu den einzelnen identifizierten Zweifelsfällen (Klärung)?

4. Bei der Klärung der Zweifelsfälle ist zu erörtern, welche Faktoren für die Korrekheit (Grammatikalität) und welche Faktoren für die Unkorrektheit (Ungrammatikalität) der Varianten sprechen. Auf der Basis einer solchen Bestimmung, die sowohl systemlinguistische als auch varietätengrammatische Aspekte zu berücksichtigen hat, wird der schwankende Status der Zweifelsfälle eindeutig bestimmbar und dadurch Orientierung ermöglicht. 
5. Bei vielen Zweifelsfällen existiert eine machtvolle Tradition der metasprachlichen

Thematisierung, die im öffentlichen Sprachbewusstsein und vermutlich auch objektsprachlich weiterwirkt. Im deutschen Sprachkontext lässt sich ein umfangreiches Textkorpus identifizieren, in dem sich spätestens seit dem 18. Jahrhundert mit sprachlichen Zweifelsfällen befasst wird. Bei der Analyse der Zweifelsfälle müssen die sozialen Konsequenzen und Voraussetzungen dieser metasprachlichen Thematisierungen mitbedacht werden.

\section{Literaturangaben}

Antos, Gerd (1996): Laien-Linguistik. Studien zu Sprach- und Kommunikationsproblemen im Alltag. Am Beispiel von Sprachratgebern und Kommunikationstrainings. Tübingen.

Beck, Götz (1996): "Sprachkritik - Sprachverfall. Zur Phänomenologie einer Sprachverwirrung". In: Peyer, Ann/Portmann, Paul R. (eds.): 61-94.

Bergmann, Rolf (1982): "Zum Anteil der Grammatiker an der Normierung der neuhochdeutschen Schriftsprache". Sprachwissenschaft 7: 261-281.

Bierwisch, Manfred (1981): "Linguistics and language error". Linguistics 19: 583-626.

Bremerich-Vos, Albert (1991): Populäre rhetorische Ratgeber. Historisch-systematische Untersuchungen. Tübingen.

Bünting, Karl-Dieter/Pospiech, Ulrike (1996): "Das Sprachtelefon: Beobachtungen von Unsicherheiten und Unzufriedenheiten von Sprachteilhabern ein Werkstattbericht". In: Böke, Karin/Jung, Matthias/Wengeler, Martin (eds.): Öffentlicher Sprachgebrauch. Praktische, theoretische und historische Perspektiven. Georg Stötzel zum 60. Geburtstag. Opladen: 121-127.

Bussmann, Hadumod (ed.) (2002): Lexikon der Sprachwissenschaft. 3. Aufl. Stuttgart.

Cherubim, Dieter (ed.) (1980): Fehlerlinguistik. Tübingen.

Cherubim, Dieter (1983): "Sprachentwicklung und Sprachkritik im 19. Jahrhundert. Beiträge zur Konstitution einer pragmatischen Sprachgeschichte." In: Cramer, Th. (ed.): Literatur und Sprache im historischen Prozess.. Bd. 2: Sprache. Tübingen: 170-188.

Cölfen, Hermann (1996): "Das Sprachtelefon an der Universität-GH - Essen. Ein Modell für ein neues Arbeitsfeld im Bereich der Linguistik?" In: Cölfen, H./Januschek, F. (eds.): Das Sprachbüro: Linguistische Beratung (...) im Spiegel der Praxisfelder: 9-21. (= OBST 53).

Coseriu, Eugenio (1988): Sprachkompetenz. Grundzüge der Theorie des Sprechens. Bearb. u. hrsg. von Heinrich Weber. Tübingen.

Davies, Winifred V. (2001): "Standardisation and the school: norm tolerance in the educational domain". Linguistische Berichte 188: 393-414.

Dieckmann, Walther (ed.) (1989): Reichthum und Armut deutscher Sprache. Reflexionen über den Zustand der deutschen Sprache im 19. Jahrhundert. Berlin/New York.

Dieckmann, Walther (1991): "Sprachwissenschaft und öffentliche Sprachdiskussion - Wurzeln ihres problematischen Verhältnisses." In: Wimmer, R. (ed.): Das 19. Jahrhundert. Sprachgeschichtliche Wurzeln des heutigen Deutsch. Berlin/New York: 355-373.

Donalies, Elke (2003): "Gebt endlich die Wortbildung frei! Über unsinnige und sinnige Kritik an der Wortbildung". Sprachreport 1: 26-32. 
Donhauser, Karin (1989): "Das Deskriptionsproblem und seine präskriptive Lösung: zur grammatikologischen Bedeutung der Vorreden in den Grammatiken des 16. bis 18. Jahrhunderts". Sprachwissenschaft 14/1: 29-57.

DUDEN Bd. 9 (2001): (Scholze-Stubenrecht, W. (Red.)) Richtiges und gutes Deutsch. Wörterbuch der sprachlichen Zweifelsfälle. 5. Aufl. Mannheim/Leipzig/Wien/Zürich.

Dückert, J./Kempcke, G. (1989): Wörterbuch der Sprachschwierigkeiten. Zweifelsfälle, Normen und Varianten im gegenwärtigen deutschen Sprachgebrauch. 3. Aufl. Leipzig.

Eisenberg, Peter (2001): "Die grammatische Integration von Fremdwörtern. Was fängt das Deutsche mit seinen Latinismen und Anglizismen an?" In: Stickel, G. (ed.): Neues und Fremdes im deutschen Wortschatz. Aktueller lexikalischer Wandel.. Berlin/New York: 183-209.

Eisenberg, Peter (2002): "Ansätze zur systematischen Beschreibung der Fremdwortorthographie. Die Gemination von Konsonantbuchstaben". In: Bommes, Michael et al. (eds.): Sprache als Form. Festschrift für Utz. Maas zum 60. Geburtstag. Wiesbaden: 121-136.

Engel, Ulrich (1988): Deutsche Grammatik. Heidelberg.

Fromkin, Victoria A. (ed.) (1980): Errors in linguistic performance. Slips of the tongue, ear, pen, and hand. New York.

Gauger, H.-M. et al. (1982): Sprachgefühl? Vier Antworten auf eine Preisfrage. Heidelberg.

Glück, Helmut (ed.) (2000): Metzler Lexikon Sprache. Stuttgart (2. Aufl.).

Greule, Albrecht/Ahlvers-Liebel, Elisabeth (1986): Germanistische Sprachpflege. Geschichte, Praxis und Zielsetzung. Darmstadt.

Greule, Albrecht (1982): "Theorie und Praxis der germanistischen Sprachpflege". Muttersprache XCII: 265-292.

Grewendorf, Günther et al. (1990): Sprachliches Wissen. Eine Einführung in moderne Theorien der grammatischen Beschreibung. 4. Aufl. Frankfurt/M.

Günther, Hartmut (1995): "Die Schrift als Modell der Lautsprache". Osnabrücker Beiträge zur Sprachtheorie (OBST) 51: 15-32.

Habermann, Mechthild (1997): "Das sogenannte 'Lutherische e'. Zum Streit um einen armen Buchstaben". Sprachwissenschaft 22/4: 435-477.

Harras, Gisela et al. (1989): Brisante Wörter von Agitation bis Zeitgeist. Ein Lexikon zum öffentlichen Sprachgebrauch. Berlin/New York.

Haß-Zumkehr, Ulrike (1995): Daniel Sanders: Aufgeklärte Germanistik im 19. Jahrhundert. Berlin/New York.

Henne, Helmut (1966): "Punktuelle und politische Sprachlenkung. Zu 13 Auflagen von Gustav Wustmanns 'Sprachdummheiten'". Zeitschrift für deutsche Sprache 21: 175-184.

Heringer, Hans Jürgen (1984): "Gebt endlich die Wortbildung frei!" Sprache und Literatur in Wissenschaft und Unterricht 53: 43-53.

Höhne, Steffen (1990): "Kommunikationsberatung Deutsch als Fremdsprache". Sprache und Literatur in Wissenschaft und Unterricht 65: 84-95.

Höhne, Steffen (1991a): "Sprachnorm und Sprachnormwandel als konstitutive Faktoren wissenschaftlicher Sprachberatung". Muttersprache 101: 193-217. 
Höhne, Steffen (1991b): "Die Rolle des Wörterbuchs in der Sprachberatung. Eine Sekundäranalyse zur Wörterbuchbenutzungsforschung". Zeitschrift für germanistische Linguistik 19: 293-321.

Jahreiß, Astrid (1990): Grammatiken und Orthographielehren aus dem Jesuitenorden. Eine Untersuchung zur Normierung der deutschen Schriftsprache in Unterrichtswerken des 18. Jahrhunderts. Heidelberg.

Jäger, Ludwig (1999): "Linguistik und Öffentlichkeit. Aspekte eines schwierigen Verhältnisses". In: Stickel, Gerhard (ed.): Sprache - Sprachwissenschaft - Öffentlichkeit. Jahrbuch 1998 des Instituts für deutsche Sprache. Berlin/New York: 243-261.

Jaeger, Christoph (1992): Probleme der syntaktischen Kongruenz. Theorie und Normvergleich im Deutschen. Tübingen.

Jakob, Karlheinz (1999): "Die Sprachnormierungen Johann Christoph Gottscheds und ihre Durchsetzung in der zweiten Hälfte des 18. Jahrhunderts". Sprachwissenschaft 24/1: 1-46.

Joeres, Rolf (1996): "Der Friede oder der Frieden. Ein Normproblem der Substantivflexion". Sprachwissenschaft 21/3: 301-336.

Keller, Rudi (1994): Sprachwandel. Von der unsichtbaren Hand in der Sprache. Zweite, überarbeitete und erweiterte Auflage. Tübingen/Basel.

Kielhöfer, Bernd (1980): Fehlerlinguistik des Fremdsprachenerwerbs. Linguistische, Lernpsychologische und didaktische Analyse von Französischfehlern. 2. Aufl. Königstein/Ts.

Klein, Wolf Peter (2000): "Prolegomena zu einer Theorie des sprachlichen Zweifelsfalls. Mit einem fremdsprachendidaktischen Ausblick." In: Klein, W.P. (ed.): Germanistik in Tallinn. Texte, Thesen und Projekte zur deutschen Sprache und Literatur. Tallinn: 60-83.

Klein, Wolfgang (1986): "Der Wahn vom Sprachverfall und andere Mythen". Zeitschrift für Linguistik und Literaturwissenschaft, Heft 62 (Sprachverfall): 11-28.

Kleppin, Karin (1998): Fehler und Fehlerkorrektur. München (= GI Fernstudieneinheit 19).

Kolde, Gottfried (1976): "Sprachberatung: Motive und Interessen der Fragensteller". Muttersprache 86 : 20-47.

Kolde, Gottfried (1980): "Sprachpflege als angewandte Sprachwissenschaft". Der Sprachdienst XXIV: 97-106.

Konopka, Marek (1996): Strittige Erscheinungen der deutschen Syntax im 18. Jahrhundert. Tübingen.

Kühn, Ingrid (2003): "Sprachberatung als Hilfeleistung im Identifikationsprozess". In: Janich, N./Thim-Mabrey, Ch. (eds.): Sprachidentität - Identität durch Sprache. Tübingen: 91-105.

Kühn, Ingrid/Almstädt, Klaus (1997): "Rufen Sie uns an - Sprachberatung zwischen Sprachwacht und Kummertelefon". Deutsche Sprache 25: 195-206.

Leuninger, Helen (1998): "Kleine Theorie des Versprechers". In: dies. Reden ist Schweigen, Silber ist Gold. Gesammelte Versprecher. 3. Aufl. München.

Lühr, Rosemarie (1992): "Gleichartigkeit, Vollständigkeit, Vermeidung von Redundanz. Prinzipien von Sprachbewertungen im 19. Jahrhundert". Muttersprache 102: 341-358.

Mackowiak, Klaus/Steffen, Karin (1991): "Statistische Auswertung der Anfragen an das Grammatische Telefon". Diskussion Deutsch 121: 518-535.

Mattheier, Klaus Jürgen (1997): "Über Destandardisierung, Umstandardisierung und Standardisierung in modernen europäischen Standardsprachen". In: Mattheier, K.J./Radtke, E. 
(eds.): Standardisierung und Destandardisierung europäischer Nationalsprachen. Frankfurt/M.: 1-9.

Meibauer, Jörg (1999): Pragmatik. Eine Einführung. Tübingen.

Müller, Wolfgang (1982): "Das Sprachgefühl auf dem Prüfstand der Philologie. Eine Materialstudie". In: Gauger, H.-M. et al.: 203-320.

Muthmann, Gustav (1994): Doppelformen in der deutschen Sprache der Gegenwart. Studie zu den Varianten in Aussprache, Schreibung, Wortbildung und Flexion. Tübingen.

Neuland, Eva (1993): "Sprachgefühl, Spracheinstellungen, Sprachbewußtsein. Zur Relevanz 'subjektiver Faktoren' für Sprachvariation und Sprachwandel". In: Mattheier, Klaus J. et al. (eds.): Vielfalt des Deutschen. Festschrift für Werner Besch. Frankfurt/M. u. a.: 723-747.

Nickisch, Reinhard M.G. (1975): Gutes Deutsch? Kritische Studien zu den maßgeblichen praktischen Stillehren der deutschen Gegenwartssprache. Göttingen.

Niederhauser, Jürg (1999): "Kaum präsente Linguistik. Zur Behandlung von Sprachfragen und sprachbezogenen Themen in der Öffentlichkeit". In: Becker-Mrotzek, M./Doppler, Ch. (eds.): Medium Sprache im Beruf. Tübingen: 37-52.

Peyer, Ann/Portmann, Paul R. (eds.) (1996): Norm, Moral und Didaktik - Die Linguistik und ihre Schmuddelkinder. Eine Aufforderung zur Diskussion. Tübingen.

Polenz, Peter von (1984): "Sprachnormung und Ansätze zur Sprachreform im Deutschen". In: Fodor, I./Hagège, C. (eds.): Language Reform. History and Future. Vol. III. Hamburg: 2352.

Polenz, Peter von (1994): Deutsche Sprachgeschichte vom Spätmittelalter bis zur Gegenwart. Bd. II: 17. und 18. Jahrhundert. Berlin/New York.

Polenz, Peter von (1999): Deutsche Sprachgeschichte vom Spätmittelalter bis zur Gegenwart. Bd. III: 19. und 20. Jahrhundert. Berlin/New York.

Püschel, Ulrich (1991): "Praktische Stilistiken - Ratgeber für gutes Deutsch?" In: Neuland, E./ Bleckwenn, H. (eds.): Stil - Stilistik - Stilisierung. Linguistische, literaturwissenschaftliche und didaktische Beiträge zur Stilforschung. Frankfurt/M. et al.: 55-68.

Reis, Marga (1979): "Ansätze zu einer realistischen Grammatik". In: Grubmüller, Klaus et al. (eds.): Befund und Deutung. Zum Verhältnis von Empirie und Interpretation in Sprachund Literaturwissenschaft. Festschrift für Hans Fromm. Tübingen: 1-21.

Sauter, Anke (2000): Eduard Engel. Literaturhistoriker, Stillehrer, Sprachreiniger. Ein Beitrag zur Geschichte des Purismus in Deutschland. Bamberg.

Scholze-Stubenrecht, Werner (1991): "Die Sprachberatungsstelle der Dudenredaktion". Deutsche Sprache 19: 178-182.

Schrodt, Richard (1995): Warum geht die deutsche Sprache immer wieder unter. Die Problematik der Werthaltungen in der deutschen Sprache. Wien.

Stötzel, Georg/Eitz, Thorsten (eds.) (2002): Zeitgeschichtliches Wörterbuch der deutschen Gegenwartssprache. Hildesheim.

Siebert-Ott, Gesa (1990): Überlegungen zum Verhältnis von Sprachberatung - Sprachförderung - Sprachunterricht am Beispiel von Kongruenzphänomenen. Köln.

WAHRIG Bd. 5 (2003): Wahrig. Fehlerfreies und gutes Deutsch. Gütersloh/München. 


\section{Quellen zur Behandlung der Zweifelsfälle ("Schwankungen", "Doppelformen") bis 1930 (chronologisch geordnet):}

Leibniz, Gottfried Wilhelm (1717/1983): Unvorgreifliche Gedanken, betreffend die Ausübung und Verbesserung der deutschen Sprache. Herausgegeben von Uwe Pörksen. Stuttgart.

Weitenauer, Ignaz (1764): Zweifel von der deutschen Sprache vorgetragen, aufgelöset, oder andern aufzulösen überlassen, sammt einem orthographischen Lexikon. Augsburg et al.

Heynatz, Johann Friedrich (1785): Anweisung zur deutschen Sprache. Berlin.

Stutz, Johann E. (1789): Kleiner Beytrag zur Beförderung deutscher Sprachrichtigkeit. Zerbst.

Angerstein, Johann Carl (1791): Anweisung, die gemeinsten Schreib- und Sprechfehler im Deutschen zu vermeiden. Für Frauenzimmer, Ungelehrte und besonders zum Gebrauch in Schulen eingerichtet. Stendal.

Heynatz, Johann Friedrich (1796): Versuch eines Deutschen Antibarbarus oder Verzeichniß solcher Wörter, deren man sich in der reinen deutschen Schreibart entweder überhaupt oder doch in gewissen Bedeutungen enthalten muß. Berlin.

Schmiedtgen, Johann G. D. (1799): Darstellung auffallender Fehler der deutschen Sprache im Umgange des gemeinen Lebens und der Mittel sie zu verbessern. Ein Seitenstück zu Heynatz Antibarbarus in Briefen. Leipzig.

Heynatz, Johann Friedrich (ed.) (1801): Neue Beiträge zur Verbesserung der Deutschen Sprache. Von einer Gesellschaft verbundener Sprachfreunde. Küstrin.

Heinsius, Theodor (1817): "Plan und Einleitung". Sprach- und Sittenanzeiger der Deutschen I /Januar 1817.

Ditscheiner, Josef Alois (1847): Neuestes und vollständiges grammatisch-orthographischstylistisches Hand- und Hilfs-Wörterbuch der deutschen Sprache mit besonderer Rücksicht auf die Schwierigkeiten, Zweifel und gangbaren Fehler in der Beugung, Fügung, Bedeutung und Schreibart der einzelnen Wörter (...). Leipzig.

Sanders, Daniel (1872): Kurzgefaßtes Wörterbuch der Hauptschwierigkeiten in der deutschen Sprache. Berlin.

Ab 1880: Wörterbuch der Hauptschwierigkeiten in der deutschen Sprache. (43./44. Aufl. 1908.) Berlin.

Grube, A.W. (1876): Streiflichter auf die Wandlungen und Schwankungen im neuhochdeutschen Sprachgebrauch. Leipzig.

Lehmann, August (1877): Sprachliche Sünden der Gegenwart. Braunschweig.

Keller, Karl Gottlieb (1879): Deutscher Antibarbarus. Beiträge zur Förderung des richtigen Gebrauchs der Muttersprache. Stuttgart.

Andresen, Karl Gustav (1880): Sprachgebrauch und Sprachrichtigkeit im Deutschen. Zitate folgen der 11. Aufl. 1923. Heilbronn..

Wolzogen, Hans von (1880): Ueber Verrottung und Errettung der Deutschen Sprache. Leipzig.

Wustmann, Gustav (1891): Allerhand Sprachdummheiten. Kleine deutsche Grammatik des Zweifelhaften, des Falschen und des Häßlichen. Ein Hilfsbuch für alle, die sich öffentlich der deutschen Sprache bedienen. 14. Aufl. 1966, zit. nach der 3. Aufl. 1903. Leipzig. 
Dr. K... (Pseudonym) (1892): Allerhand Sprachverstand. Kleine deutsche Sprachlehre für Alle, denen ihr deutsches Sprachgefühl am Herzen liegt. Bonn.

Matthias, Theodor (1892): Sprachleben und Sprachschäden. Ein Führer durch die Schwankungen und Schwierigkeiten des deutschen Sprachgebrauchs. 6. Aufl. 1930. 1896 auch für "Schüler" überarbeitet erschienen. Leipzig.

Kaerger, Karl (1892): In tyrannunculos. Streitschrift zur Vertheidigung der deutschen Sprachfreiheit. Berlin.

Minor, Jakob (1892): Allerhand Sprachgrobheiten. Eine höfliche Entgegnung. Stuttgart.

Brunner, Armin (1895): Schlecht Deutsch. Eine lustige und lehrreiche Kritik unserer neuhochdeutschen Mundunarten. Wien/Leipzig.

Matthias, Theodor (1896): Kleiner Wegweiser durch die Schwankungen und Schwierigkeiten des deutschen Sprachgebrauchs. Überarbeitung für den Schulgebrauch von Matthias 1892. Leipzig.

Matthias, Theodor (1897): Aufsatzsünden. Warnende Beispiele zu Nutz und Frommen der deutschen Schuljugend und zur Ersparung vieler roter Tinte. Leipzig.

Poetzsch, W. (1897): Rez. von Matthias 1896 und Matthias 1897. Pädagogisches Archiv 39: 814-826.

Bennewitz, Alexander (1898): Die Schwierigkeiten unserer Muttersprache. Übersichtliche Zusammenstellung der zweifelhaften Fälle im mündlichen und schriftlichen Sprachgebrauche, mit besonderer Berücksichtigung der kaufmännischen Sprache. 4. Aufl. 1920. Leipzig.

Contze, Heinrich (1898): Ueber den Gebrauch des Pronomens derselbe mit besonderer Ruecksicht auf die von Wustmann Allerhand Sprachdummheiten erhobenen Bedenken. Herford.

Tappolet, Ernst (1898): Wustmann und die Sprachissenschaft. Zürich.

Franke, Theodor (1903): Schwierigkeiten und Schwankungen des deutschen Sprachgebrauchs. Hilfsbuch für den deutschen Sprachunterricht. Dresden.

Fischer, Paul (1903): Über Schwankungen im deutschen Sprachgebrauche der Gegenwart. Stettin.

Schmeitzner, Werner (1909): Die deutsche Sprache und ihre Verbesserer Harden und Wustmann. Leipzig.

Briegleb, Otto (1911): Wider die Sprachverderbnis. Ein Beitrag zur Wahrung des Standes der deutschen Sprache. Mit einem Wortverzeichnis. Leipzig.

Engel, Eduard (1918): Gutes Deutsch. Ein Führer durch Falsch und Richtig. Zitiert nach der 4. Aufl. 1929. Leipzig.

Schneider, Karl (1930): Was ist Gutes Deutsch? Ein Führer durch die Schwierigkeiten und Zweifelsfälle des heutigen deutschen Sprachgebrauchs. 2. Aufl. 1931. München. 